\title{
Elastically Shaped Wing Optimization and Aircraft Concept for Improved Cruise Efficiency
}

\author{
Nhan Nguyen* \\ NASA Ames Research Center, Moffett Field, CA 94035 \\ Khanh Trinh ${ }^{\dagger}$ \\ Stinger Ghaffarian Technologies Inc., Moffett Field, CA 94035 \\ Kevin Reynolds ${ }^{\ddagger}$ \\ NASA Ames Research Center, Moffett Field, CA 94035 \\ James Kless ${ }^{\S}$ \\ Science \& Technology Corp., Moffett Field, CA 94035 \\ Michael Aftosmis ${ }^{\text {It }}$ \\ NASA Ames Research Center, Moffett Field, CA 94035 \\ James Urnes, Sr.\| \\ Boeing Research \& Technology, St. Louis, MO 63166 \\ Corey Ippolito** \\ NASA Ames Research Center, Moffett Field, CA 94035
}

\begin{abstract}
This paper presents the findings of a study conducted in 2010 by the NASA Innovation Fund Award project entitled "Elastically Shaped Future Air Vehicle Concept". The study presents three themes in support of meeting national and global aviation challenges of reducing fuel burn for present and future aviation systems. The first theme addresses the drag reduction goal through innovative vehicle configurations via non-planar wing optimization. Two wing candidate concepts have been identified from the wing optimization: a drooped wing shape and an inflected wing shape. The drooped wing shape is a truly biologically inspired wing concept that mimics a seagull wing and could achieve about $5 \%$ to $6 \%$ drag reduction, which is aerodynamically significant. From a practical perspective, this concept would require new radical changes to the current aircraft development capabilities for new vehicles with futuristic-looking wings such as this concept. The inflected wing concepts could achieve between $3 \%$ to $4 \%$ drag reduction. While the drag reduction benefit may be less, the inflected-wing concept could have a near-term impact since this concept could be developed within the current aircraft development capabilities. The second theme addresses the drag reduction goal through a new concept of elastic wing shaping control. By aeroelastically tailoring the wing shape with active control to maintain optimal aerodynamics, a significant drag reduction benefit could be realized. A significant reduction in fuel burn for long-range cruise from elastic wing shaping control could be realized. To realize the potential of the elastic wing shaping control concept, the third theme emerges that addresses the drag reduction goal through a new aerodynamic control effector called a variable camber continuous trailing edge flap. Conventional aerodynamic control surfaces are discrete independent surfaces that cause geometric discontinuities at the trailing edge region. These discontinuities promote vorticities which result in drag rises as well as noise sources. The variable camber trailing edge flap concept could provide a substantial drag reduction benefit over a conventional discrete flap system. Aerodynamic simulations show a drag reduction of over $50 \%$ could be achieved with the flap concept over a conventional discrete flap system.
\end{abstract}

\footnotetext{
*AIAA Associate Fellow, Intelligent Systems Division, nhan.t.nguyen@nasa.gov

†Intelligent Systems Division, khanh.v.trinh@nasa.gov

${ }^{\ddagger}$ Intelligent Systems Division, kevin.w.reynolds@nasa.gov

\$Systems Analysis Branch, james.kless-1@nasa.gov

MApplied Modeling and Simulation Branch, michael.aftosmis@nasa.gov

"Program Manager, Platform Systems/Subsystems Technology, james.m.urnes-sr@boeing.com

** Intelligent Systems Division, corey.a.ippolito@nasa.gov
} 


\section{Introduction}

NASA is conducting research that focuses on new aeronautic technologies that could potentially revolutionize aviation systems that could lead to improved aerodynamic efficiency, less fuel burn, and reduced noise and emissions. These important goals represent current challenges in the present aviation systems in response to the emerging needs for innovative aircraft design that can address future aviation systems. In the context of commercial aviation, civilian aircraft remains the largest U.S. export category (\$9.4 billion, "U.S. Export Fact Sheet, March 2009). The increasing demand for fuel-efficient aircraft for global commerce prompts the aircraft industry to address improved fuel efficiency as a top national and global challenge.

This paper introduces a multi-disciplinary vehicle design optimization concept, based on a four-month study conducted in 2010 by the NASA Innovation Funds Award Project entitled "Elastically Shaped Future Air Vehicle Concept" $[1,2]$. The project was participated by NASA Ames Research Center, NASA Langley Research Center, Boeing Research \& Technology, and California State University, Sacramento. The concept represents an innovative approach that holds potential to help realize the goal of drag reduction that can directly address the global challenge of improving aircraft fuel efficiency.

Aircraft are typically designed to maintain sufficient structural rigidity for safe load-carrying capacity. Modern engineered materials such as composites have begun to appear in new airframe designs that can provide less structural rigidity while maintaining the same load-carrying capacity. A good example of a current airframe design that leverages light-weight composites materials is the Boeing 787 Dreamliner aircraft, which exhibits a greater degree of wing flexibility than an older-generation aircraft design. Thus, there is a realization that future aircraft concepts can be developed to take advantage of the structural flexibility afforded by modern engineered materials to improve aerodynamic efficiency.

Elastically shaped aircraft is a concept whereby highly flexible aerodynamic surfaces are elastically shaped inflight by actively controlling the wing wash-out twist and wing bending deflection in order to change the local angle of attack in such a manner that can result in lower fuel burn by drag reduction during cruise and or enhanced performance during take-off and landing by increasing lift. Moreover, structural flexibility will be leveraged to realize a revolutionary, optimal non-planar wing shape design that can accommodate a significant curvature for drag reduction benefits as opposed to a conventional planar wing design. Elastically shaped aircraft, therefore, may be viewed as a biologically-inspired concept that could potentially revolutionize the conventional airframe design. Taking a cue from birds' efficient shape-changing wings, this concept may be able to bring future aircraft concepts to the next level in terms of performance, efficiency, and maneuverability.

To realize the goals of this proposal, this project conducts a feasibility study in 2010 with the funding support of NASA Innovation Fund Award sponsored by NASA Innovative Partnerships Program. The plan of this study is to conduct a multi-disciplinary design, analysis and optimization to examine the potential benefits of the elastically shaped future aircraft concept over a conventional vehicle design. The four major technical areas of the study are:

1. Vehicle conceptual design and optimization - A futuristic elastically shaped wing superimposed on an available commercial transport fuselage will be designed using aerodynamic strip theory and vortex lattice aerodynamic code. Optimization will be conducted to identify an optimal shape defined by the varying curvature and washout twist of the elastic wing that minimizes induced drag or maximizes lift-to-drag ratio, hence aircraft range. A comparative study of the fuel savings will be made using available performance data for a representative commercial transport and engines.

2. Aeroelastic flight dynamic modeling - A static and dynamic aeroservoelastic model of the elastically shaped wing will be developed in conjunction with a flight dynamic model for analyzing aerodynamics, stability and control of the elastically shaped aircraft.

3. Elastically wing shaping actuation design - A low drag distributed actuation concept will be developed to size and strategically place new aerodynamic surfaces throughout the elastically shaped wing. The distributed control surfaces will be used to actively shape the elastic wing to gain aerodynamic efficiency. Commercially available sensor technology for shape measurement will be leveraged and assumed to be available for elastically wing shaping control.

4. Flight control design and vehicle simulation - A multi-objective flight control system will be developed to simultaneously gain aerodynamic efficiency and maintain traditional pilot command-tracking tasks for guidance and navigation. A guidance law to achieve a low drag objective will be developed for a cruise phase to specify 
both the desired drag and vehicle attitude objectives for flight control performance. Multi-objective optimal control will be introduced for flight control design. A complete vehicle control simulation will be performed to demonstrate feasibility.

This paper will mainly focus on the results of the technical areas 1 and 3 .

\section{Vehicle Aerodynamic Optimization}

The goal of this study is to develop new wing shape concepts that will provide aerodynamic benefits of induced drag reduction. To conduct this study, aerodynamic optimization is performed to identify optimal wing shapes that can achieve induced drag reduction. In addition, other ideas of drag reduction are explored besides the wing shape optimization. The ultimate goal of this project is to develop novel aircraft concepts based on optimized wing shapes and other ideas obtained from this study that could potentially provide a breakthrough from the equilibrium in the current aircraft design which has remained virtually unchanged for the past several decades. These new concepts could provide a new impetus to aircraft design for future aviation systems that would operate in a resource-constrained ecosystem.

\subsection{Baseline Vehicle}

The baseline vehicle selected for the study is a notional single-aisle, mid-size, 200-passenger aircraft. The geometry of the baseline aircraft is obtained by scaling up the geometry of NASA generic transport model (GTM) by a scale of 200:11. The GTM is a research platform that includes a wind tunnel model and a remotely piloted vehicle, as shown in Figure 1 [3]. Figure 2 is an illustration of the baseline aircraft. The reason for selecting this baseline vehicle is that there already exists an extensive wind tunnel aerodynamic database that would be used subsequently in the study for validating results of the optimization study. The baseline configuration represents one of the most common types of transport aircraft in the commercial aviation sector that provides short-to-medium range passenger carrying capacities.

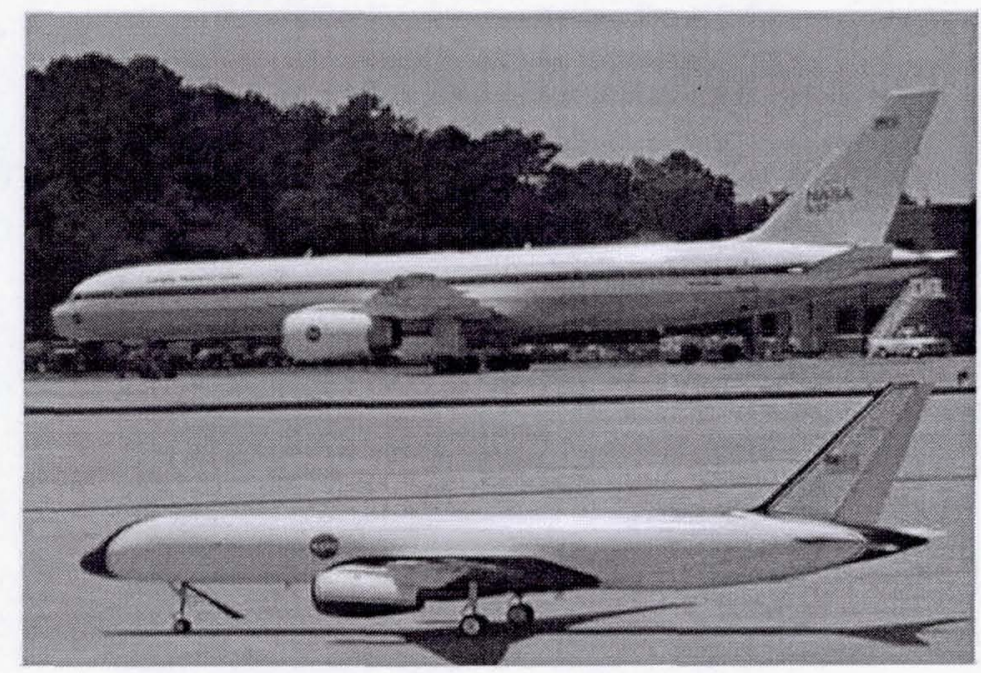

Figure 1 - Generic Transport Model (GTM) Remotely Piloted Vehicle at NASA Langley 


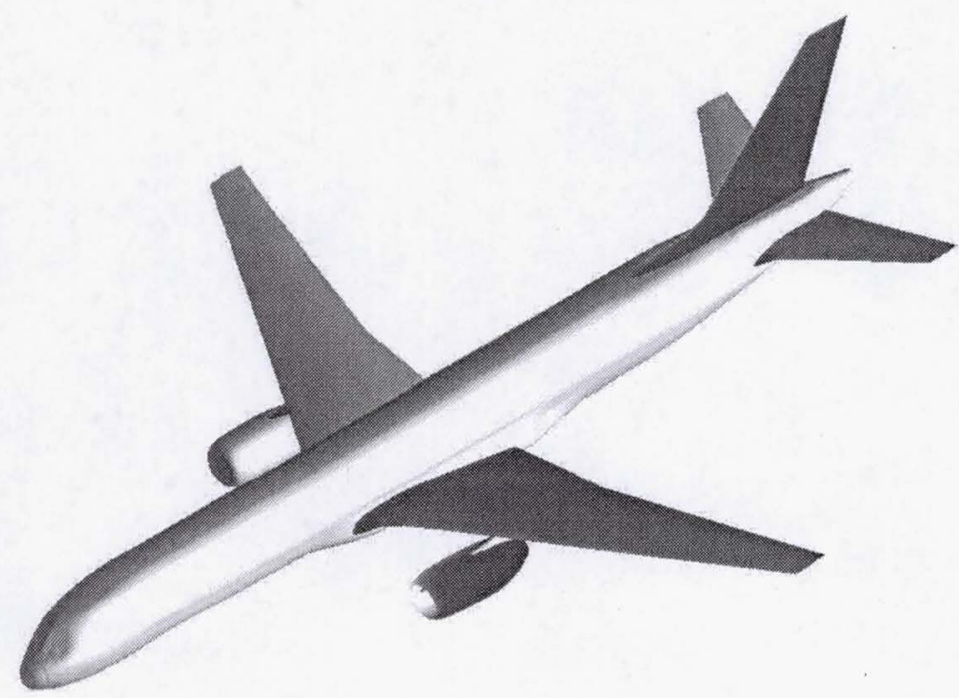

Figure 2 - Baseline Aircraft

The aircraft has a take-off weight of 200,000 lbs for a typical operating load (gear up, flap up) that includes cargo, fuel, and passengers. The operational empty weight (OEW) of the aircraft is 132,821 lbs. Fuel weighs about 50,000 lbs for a range of about 3,000 nautical miles. Table 1 shows some typical aircraft characteristics:

\begin{tabular}{|c|c|}
\hline Wing Reference Area, $S_{\text {ref }}$ & $1951 \mathrm{ft}^{2}$ \\
\hline Aspect Ratio, $A R$ & 7.82 \\
\hline Taper Ratio, $\lambda$ & 0.212 \\
\hline MAC, $\bar{c}$ & $16.6417 \mathrm{ft}$ \\
\hline Wing Span, $b$ & $124.8333 \mathrm{ft}$ \\
\hline Wing Leading Edge Sweep Angle, $\Lambda$ & $28.4286^{\circ}$ \\
\hline Wing Dihedral Angle, $\Gamma$ & $5^{\circ}$ \\
\hline
\end{tabular}

Table 1 - Baseline Aircraft Characteristics

To compute the mass and inertia properties of the baseline aircraft, a component-based approach is used. The aircraft is divided into the following components: fuselage, wings, horizontal tails, vertical tail, engines, operational empty weight (OEW) equipment, and typical load including passengers, cargo, and fuel. Based on [4], an average wing mass relative to the total empty weight of the aircraft is taken to be $24.2 \%$ of the OEW. Similarly, the mass contributions of the fuselage and the combined horizontal and vertical tails are taken to be $19.0 \%$ and $4.2 \%$, respectively.

The aircraft is powered by two $44,000-1 \mathrm{~b}$ rated turbofan engines. The maximum thrust per engine at sea level takeoff is 44,000 lbs and varies linearly to 30,000 lbs at sea level Mach 0.8 The thrust specific fuel consumption (TSFC) at sea level take-off is $0.3090 / \mathrm{hr}$ and varies linearly to $0.5943 / \mathrm{hr}$ at sea level Mach 0.8 . The total maximum engine thrust and TSFC as functions of altitude and Mach number are given by the following formulas:

$$
\begin{gathered}
T_{\max }(h, M)=(-77031 M+100986)\left(1+\frac{\gamma-1}{2} M^{2}\right)^{\frac{\gamma}{\gamma-1}} \delta(h) \\
c(M, h)=(0.40756 M+0.23356) \sqrt{\theta(h)\left(1+\frac{\gamma-1}{2} M^{2}\right)}
\end{gathered}
$$

where $T_{\max }$ is the maximum thrust, $c$ is the TSFC, $M$ is Mach number, $\delta=p / p_{S L}$ is the pressure ratio, $\theta=T / T_{S L}$ is the temperature ratio, and $p_{S L}$ and $T_{S L}$ are the pressure and temperature at sea level.

The TSFC represents the fuel burn rate, thus an important parameter in the estimation of range and fuel burn. 


\subsection{Computational Aerodynamic Tools}

Two computational tools are used in the study. A vortex-lattice computational code, called VORVIEW, is used extensively in the design, analysis, and optimization for the development of elastically shaped aircraft concepts [6]. Another computational fluid dynamic (CFD) tool, Cart3D, is used for validation of candidate optimized vehicle concepts identified by the optimization study [7]. Both of these tools have been used in the past for modeling the NASA GTM. Therefore, some amount of validation has been done previously to compare the computational results of these two codes with wind tunnel data.

VORVIEW provides a rapid method for estimating aerodynamic force and moment coefficients as well as aerodynamic stability and control derivatives of a given aircraft configuration. It is based on the vortex-lattice aerodynamic theory. The vehicle configuration is constructed within VORVIEW by a series of panels that are formed by spanwise and chordwise locations of bound vortices. VORVIEW computes the vehicle aerodynamics in both the longitudinal and lateral directions independently. The longitudinal and lateral aerodynamics are then combined to produce overall aerodynamic characteristics of the vehicle at any arbitrary angle of attack and angle of sideslip. Due to the inviscid nature of any vortex-lattice method, the drag prediction by VORVIEW is most reliable for induced drag prediction. Viscous drag and wave drag are not computed, but a skin friction drag correction can be implemented directly in VORVIEW.

Cart3D is a high-fidelity, three-dimensional Euler solver suitable for CFD analysis on complex geometries. Cart3D has a mesh adaptation feature that uses adjoint-weighted residual error estimates to drive mesh adaptation for user selected outputs (such as drag or lift). In this way, the mesh refinement procedure generates a mesh that reduces the discretization errors in the outputs so that the influence of these errors on the output functional is below a specified error tolerance. A more complete analysis of the adjoint formulation and its applications on complex geometries can be found in [8] and [9]. Another valuable capability of Cart3D is the ability to achieve a user-requested value of $C_{L}$ by modifying the angle of attack at specified time steps. This capability can be helpful in comparing induced-drag benefits across different geometries.

Previously, both VORVIEW and Cart3D had been used to model the GTM geometry with various damages to aerodynamic surfaces for the NASA Damage Adaptive Control System (DACS) project under the NASA Aviation Safety and Security program in 2005 [5]. Wind tunnel data for the 5.5\% model of the GTM were obtained in the 14-by 22 -foot low speed subsonic wind tunnel at NASA Langley. The test section Mach number is fixed at 0.084 which is well within the incompressible flow regime. Force and moment data were collected over a wide range of angle of attack and angle of sideslip. Computational results from VORVIEW and Cart3D were subsequently compared with wind tunnel data to assess relative accuracy of the aerodynamic prediction. Due to a convergence issue with Cart3D which caused a substantial difference with the wind tunnel data, the Mach number for Cart3D was increased to 0.2 to improve the solution convergence while VORVIEW results were for Mach number of 0.084 . Figures 3 and 4 shows the comparison of the $C_{L}$ and $C_{D}$ values versus the angle of attack at zero sideslip angle [5].

VORVIEW lift prediction matched wind tunnel data very well up to almost $\alpha=10^{\circ}$. Cart3D seemed to overpredict the lift coefficient. However, this could be due to Cart3D results computed for a higher Mach number than both VORVIEW results and wind tunnel data. Both VORVIEW and Cart3D produced a very similar drag prediction for incompressible flow, but they were lower than the measured drag in the wind tunnel. The measured drag in the wind tunnel is not corrected for a full-scale Reynold number at cruise. When a Reynolds number correction is applied, the $C_{D}$ coefficient should decrease in theory as the Reynolds number increases. The discrepancy could also arise from the lack of viscous loss models in both VORVIEW and Cart3D results. 


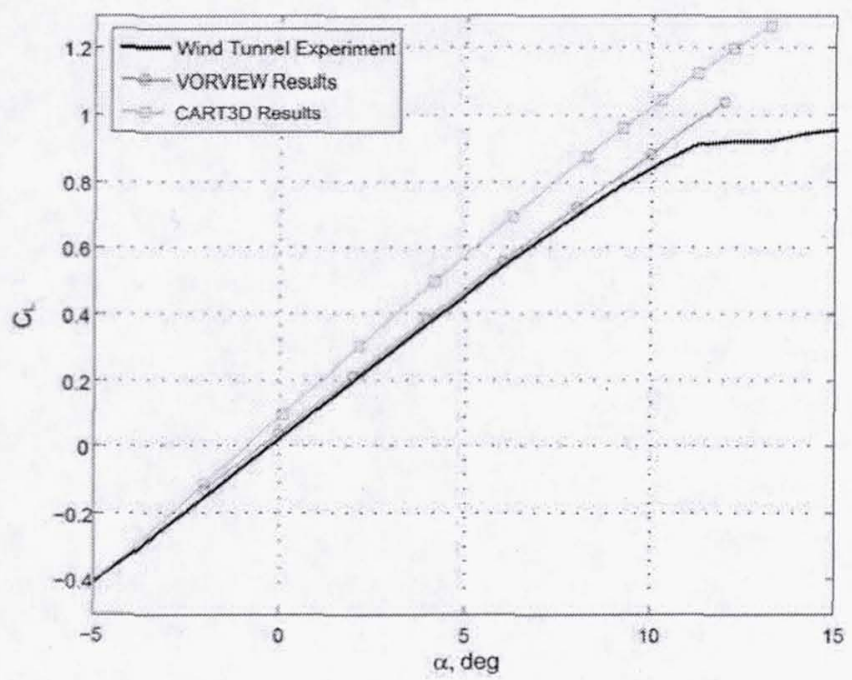

Figure $3-C_{L}$ vs. $\alpha$ Comparison

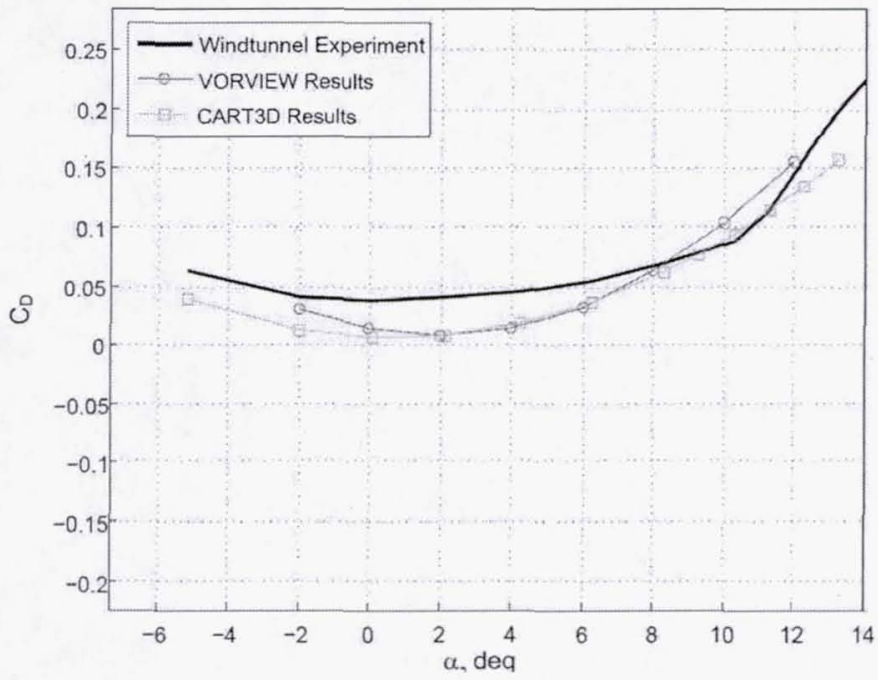

Figure $4-C_{D}$ vs. $\alpha$ Comparison

\subsection{Automated Vehicle Geometry Modeling Tool}

Aircraft configuration optimization requires an efficient way to generate new vehicle configurations during the optimization. An automated vehicle geometry modeling tool has been developed in MATLAB specifically for the optimization study. The vehicle geometry modeler directly outputs a geometry input file that can be read by VORVIEW during the optimization. The vehicle geometry modeler has access to the outer mold line geometry information of the full-scale GTM. The coordinate reference frame $\left(x_{V}, y_{V}, z_{V}\right)$ defines the coordinate system used in the vehicle geometry model. Wing chordwise and flapwise bending deflection shapes and a twist distribution are superimposed on top of the wing geometry as shown in Figure 5. A new wing geometry is generated by performing successive coordinate translation and rotation. The order of the coordinate transformation is important. To reduce the frontal area of the wing due to twist which can affect drag, the order of the coordinate transformation is as follows: 
1. A coordinate rotation to account for twist is performed first by rotating a baseline wing section about its area center by a specified twist angle at a given $y_{V}$-coordinate. The transformed coordinates due to twist are computed as

$$
\begin{aligned}
& x_{V}^{\prime}=\bar{x}+\left(x_{V}-\bar{x}\right) \cos \Theta\left(y_{V}\right)-\left(z_{V}-\bar{z}\right) \sin \Theta\left(y_{V}\right) \\
& z_{V}^{\prime}=\bar{z}+\left(x_{V}-\bar{x}\right) \sin \Theta\left(y_{V}\right)+\left(z_{V}-\bar{z}\right) \cos \Theta\left(y_{V}\right)
\end{aligned}
$$

where $\Theta$ is the twist angle, positive nose-down and negative nose-up.

2. A coordinate translation in the $x_{V}$-direction to account for chordwise bending is performed next by translating the previously transformed $x_{V}^{\prime}$-coordinate by a specified chordwise bending deflection at a given $y_{V}$-coordinate. The transformed coordinates due to chordwise bending are computed as

$$
\begin{gathered}
x_{V}^{\prime \prime}=x_{V}^{\prime}+V\left(y_{V}\right) \\
z_{V}^{\prime \prime}=z_{V}^{\prime}
\end{gathered}
$$

where $V$ is the chordwise bending deflection, positive swept back and negative swept forward.

3. Finally, a coordinate translation in the $z_{V}$-direction to account for flapwise bending is performed by translating the previously transformed $z_{V}^{\prime \prime}$ by a specified flapwise bending deflection at a given $y_{V}$-coordinate. The transformed coordinates due to flapwise bending are computed as

$$
\begin{gathered}
x_{V}^{\prime \prime \prime}=x_{V}^{\prime \prime} \\
z_{V}^{\prime \prime \prime}=z_{V}^{\prime \prime}+W\left(y_{V}\right)
\end{gathered}
$$

where $W$ is the flapwise bending deflection, positive up and negative down.

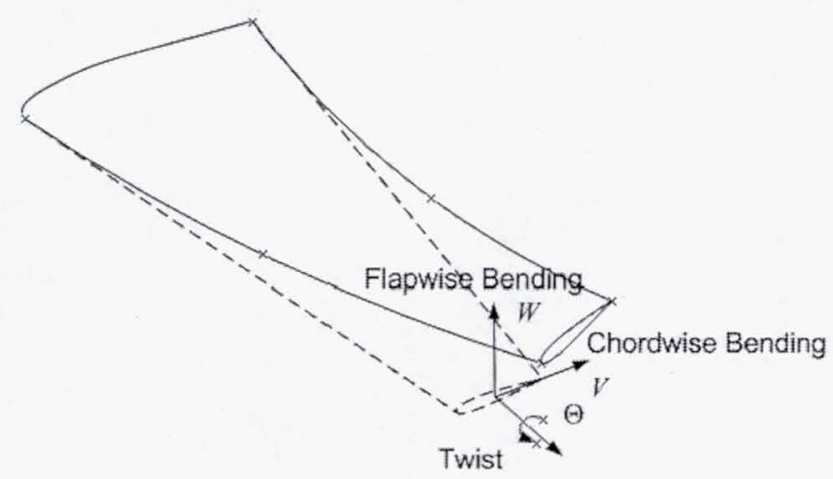

Figure 5 - Wing Bending Deflections and Twist

It is noted that the coordinate transformation is not length-preserving since as the curvature increases, the wing span also increases. The increase in the wing span can be computed as

$$
\Delta b=2 \int_{y_{r}}^{y_{t}}\left(\sqrt{1+\left[V^{\prime}\left(y_{V}\right)\right]^{2}+\left[W^{\prime}\left(y_{V}\right)\right]^{2}}-1\right) d y \approx \int_{y_{r}}^{y_{t}}\left\{\left[V^{\prime}\left(y_{V}\right)\right]^{2}+\left[W^{\prime}\left(y_{V}\right)\right]^{2}\right\} d y
$$

The engine geometry and the pylon geometry are also affected by the coordinate transformation of the wing geometry. Thus, coordinate transformations of the engine geometry and the pylon geometry are also performed in order to keep the relative positions of the engines and pylons with respect to the wing geometry the same at the engine mount locations on the wings.

The vehicle geometry also includes the following additional features:

- A "squashed" fuselage geometry can be modeled by scaling the $y_{V}$ and $z_{V}$-coordinates of the fuselage by specified scaling factors. The squashed fuselage concept is of particular interest, since it can provide an additional lift contribution derived from the fuselage itself. As a result, the wing lift is reduced that results in lower lift-induced drag. The squashed fuselage concept will be discussed in the subsequent section on optimization. 
- A "high-wing" geometry allows the wing position to be placed above the fuselage centerline. The majority of commercial aircraft are of low-wing configurations which provide added roll stability with a positive wing dihedral. Many military transports such as C-5 are designed with a high-wing configuration.

- A "V-tail" geometry is available that allows the V-tails to serve as both directional stabilizer and horizontal stabilizer. This can be an advantage if the wing curvature is significant that can also contribute to the directional stability, thereby allowing the vertical tail to be eliminated for weight savings.

- A new low-drag continuous trailing edge (TE) flap concept has been developed as part of this study and is also included in the vehicle geometry model. The benefit of drag reduction due to this flap concept will be discussed later.

The ability of the geometric modeler to superimpose bending deflection shapes and twist on a rigid wing shape provide a future capability for developing a coupled aeroelastic-aerodynamic modeling tool by coupling an aerodynamic code such as VORVIEW with a structural analysis code that computes bending deflection shapes and twist. This capability would fill a gap that is highly desirable and would greatly benefit studies like the present one.

Figure 6 illustrates various vehicle concepts generated by the automated vehicle geometry modeler.
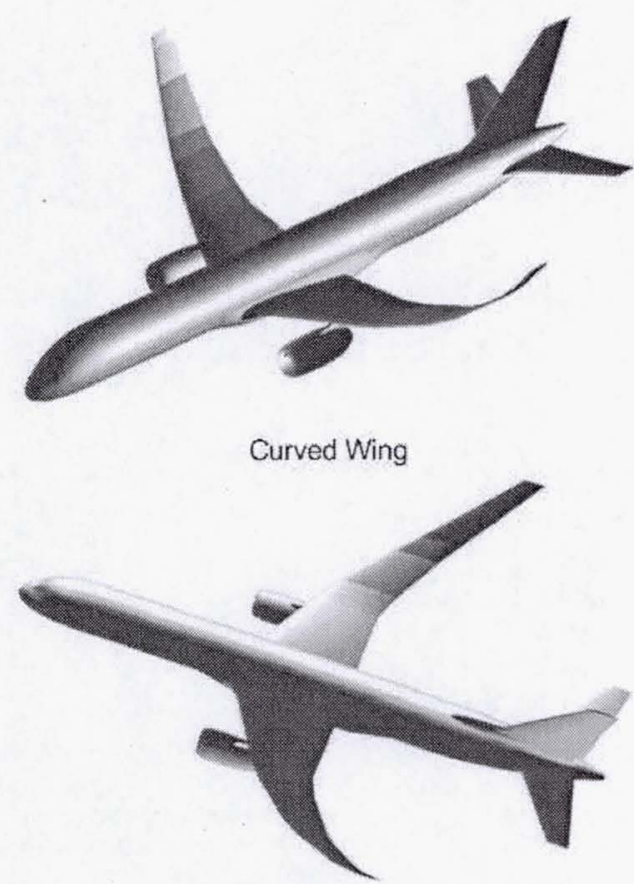

Curved Swept Back Wing

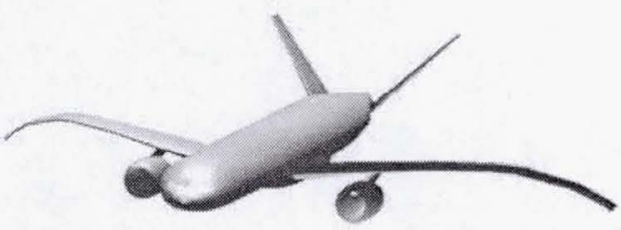

V-Tail

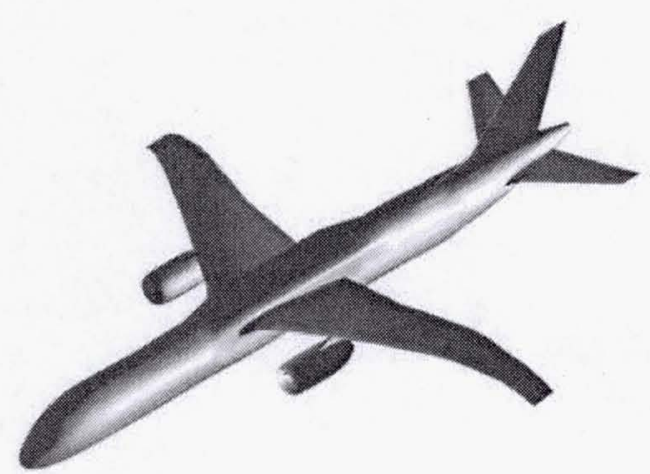

High Wing

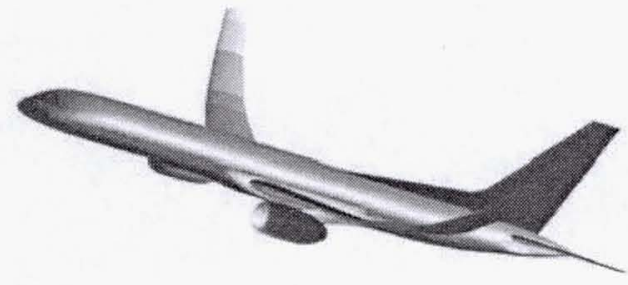

Squashed Fuselage

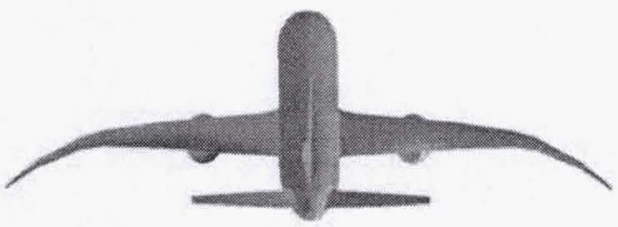

Continuous TE Flap

Figure 6 - Automated Vehicle Geometry Modeling 


\subsection{Vehicle Design Optimization Approach}

An aerodynamic optimization is conducted to develop candidate elastically shaped aircraft concepts that achieve lower drag than the baseline vehicle. The focus of the design optimization is a new wing geometry that replaces the conventional wing of the baseline vehicle. Any potential benefit of other geometric features such as squashed fuselage or V-tail may be additive to the new wing geometry. Thus, these new features are not part of the optimization.

Given that the use of modern light weight composites materials is becoming more prevalent in modern aircraft due to the benefit of weight savings while providing sufficient load carrying capacities, the conventional straight wing design might give way to a new type of wings that could include significant curvatures and flexibility that could be tailored for improved aerodynamic efficiency. Thus, the goal of the optimization is to allow each wing section to possess all three degrees of freedom in chordwise displacement, flapwise displacement, and twist. These degrees of freedom then would become the design variable in the optimization. In theory, this could be done. However, a simpler approach is pursued in the interest of time. This approach parametrizes the wing degrees of freedom by using assumed shape functions with unknown coefficients.

In particular, each shape function is described by a 4th degree polynomial with 5 unknown coefficients or design variables. Moreover, the wing sections inboard of BBL $21.6000 \mathrm{ft}$ at the engine section are assumed to be unaffected due to the large structural stiffness near the wing root that could present a challenge to shape a wing. Thus, the shape function starts at BBL $21.6000 \mathrm{ft}$. Furthermore, the displacement and slope of the shape function are enforced to be zero at this location. This enforcement reduces the number of design variables from 5 to 3 . A further simplification is made by eliminating the chordwise displacement components. Thus, the shape functions are given by

$$
\begin{aligned}
& W\left(y_{V}\right)= \begin{cases}a_{4}\left(\frac{y_{V}-y_{e}}{y_{t}-y_{e}}\right)^{4}+a_{3}\left(\frac{y_{V}-y_{e}}{y_{t}-y_{e}}\right)^{3}+a_{2}\left(\frac{y_{V}-y_{e}}{y_{t}-y_{e}}\right)^{2} & y_{e}<y_{V} \leq y_{t} \\
0 & y_{V} \leq y_{e}\end{cases} \\
& \Theta\left(y_{V}\right)= \begin{cases}b_{4}\left(\frac{y_{V}-y_{e}}{y_{t}-y_{e}}\right)^{4}+b_{3}\left(\frac{y_{V}-y_{e}}{y_{t}-y_{e}}\right)^{3}+b_{2}\left(\frac{y_{V}-y_{e}}{y_{t}-y_{e}}\right)^{2} & y_{e}<y_{V} \leq y_{t} \\
0 & y_{V} \leq y_{e}\end{cases}
\end{aligned}
$$

where $y_{e}=21.6000 \mathrm{ft}$ and $y_{t}=62.1286 \mathrm{ft}$ which is the BBL of the wing tip.

The shape function optimization thus becomes a parametric optimization where the design variables are $a_{i}$ and $b_{i}, i=2,3,4$. Upper and lower limits on the shape functions are imposed as constraints on the flapwise bending displacement and twist at the wing tip such that

$$
\begin{aligned}
& \left|W\left(y_{t}\right)\right|=\left|a_{4}+a_{3}+a_{2}\right| \leq W_{t, \max } \\
& \left|\Theta\left(y_{t}\right)\right|=\left|b_{4}+b_{3}+b_{2}\right| \leq \Theta_{t, \max }
\end{aligned}
$$

where $W_{t, \max }=20 \mathrm{ft}$ is the maximum allowable flapwise bending displacement and $\Theta_{t, \max }=4.5^{\circ}$ is the maximum allowable twist at the wing tip.

Moreover, an additional constraint is imposed on the optimization and that is the cruise condition. For a given vehicle weight flying at a given airspeed and altitude, there corresponds a lift coefficient that the vehicle must operate at. Thus, the cruise condition is expressed as

$$
C_{L}=\frac{W}{q_{\infty} S}
$$

The design point for the baseline aircraft is selected for an operating weight of $W=200,000 \mathrm{lbs}$, cruise speed of $M_{\infty}=0.8$, and cruise altitude at $30,000 \mathrm{ft}$. The design cruise lift coefficient is $C_{L}=0.364$. This design lift coefficient is enforced during the optimization.

The optimization is conducted using a sampling method over a chosen design space. The design space is chosen such that each design variable $a_{i}$ and $b_{i}, i=2,3,4$, can take on any one of three pre-selected values as shown in Table 2 such that the wing tip constraints are satisfied.

\begin{tabular}{|c|c|c|c|c|c|}
\hline$a_{4}$ & $a_{3}$ & $a_{2}$ & $b_{4}$ & $b_{3}$ & $b_{2}$ \\
\hline $0, \pm 7.5$ & $0, \pm 3.75$ & $0, \pm 8.75$ & $0, \pm 1.5$ & $0, \pm 1.5$ & $0, \pm 1.5$ \\
\hline
\end{tabular}

Table 2 - Design Space for Parametric Optimization 
This is a sample of 729 possible shape functions or design points, which is fairly limited due to the time constraint. All the possible shape functions are plotted in Figures 6 and 7 . Future work will investigate expanding the design space that will include a larger number of sampling points.

To implement the optimization, a computer code is developed to couple the vehicle geometry modeler with the aerodynamic code VORVIEW. Each design point is evaluated with a different combination of the parameters $a_{i}$ and $b_{i}, i=2,3,4$. The execution time for each design point in VORVIEW is about 2 minutes on a single dual-core CPU computer. Thus, each optimization cycle takes about one day to complete.

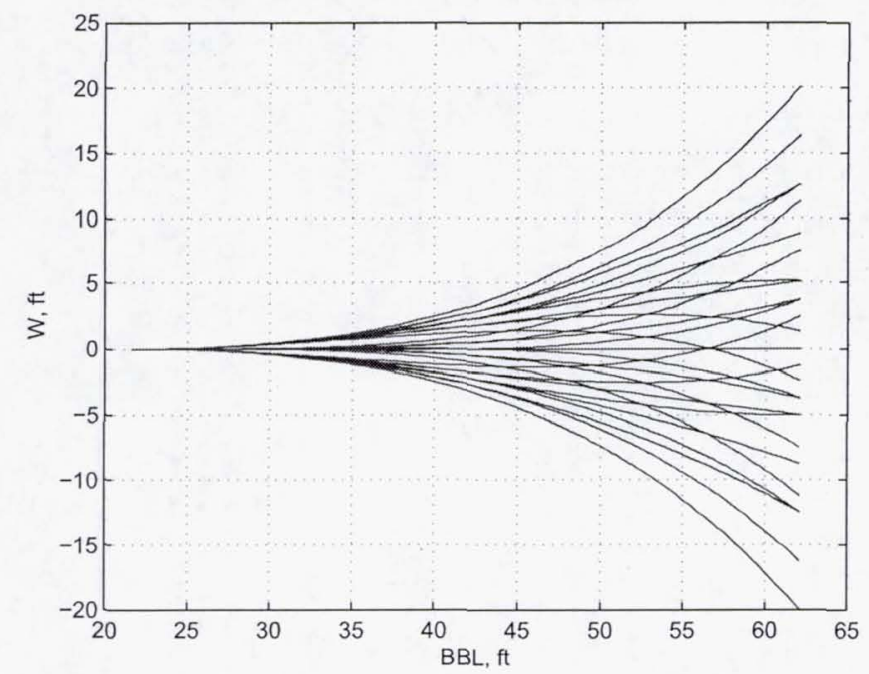

Figure 7 - Bending Shape Function Sample

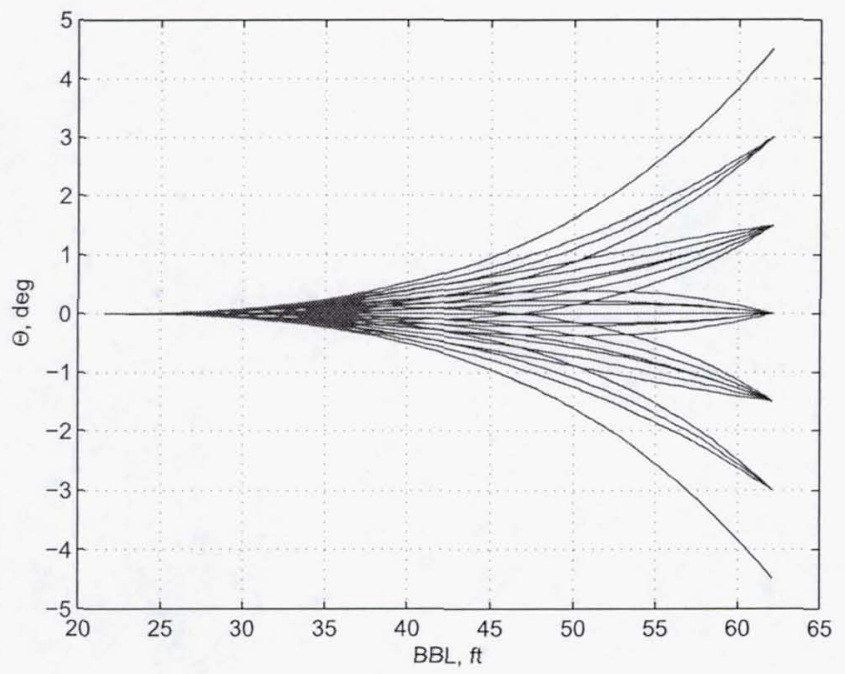

Figure 8 - Torsion Shape Function Sample

\subsection{Optimization Results}

The drag coefficient of the baseline aircraft at the design flight condition as computed by VORVIEW is $C_{D}=0.010$. At each design point, the $C_{D}$ value corresponding to the design cruise $C_{L}=0.364$ is obtained. The results of the $C_{D}$ 
calculation from the optimization are plotted in Figure 9. Each point on the plot corresponds to a given design. As can be seen, there are several designs with $C_{D}$ less than that of the baseline aircraft.

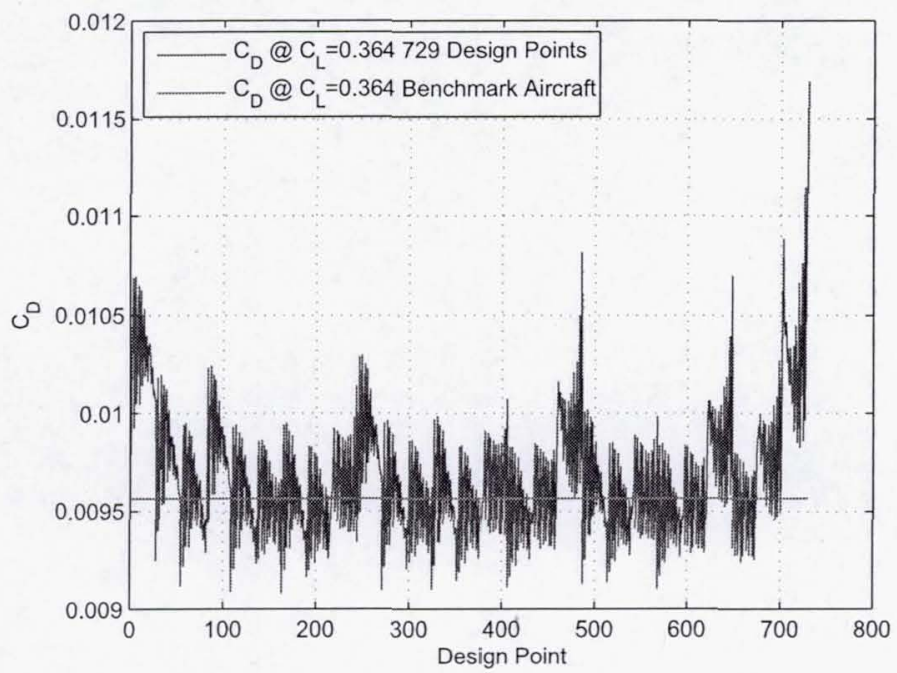

Figure $9-C_{D}$ Variation in Design Space

\subsubsection{Drooped Wing Shape}

The design corresponding to the minimum $C_{D}$ is identified as a vehicle with a drooped wing shape, which has a significant negative wing curvature with the wing tip at its maximum negative deflection as shown in Figure 10.
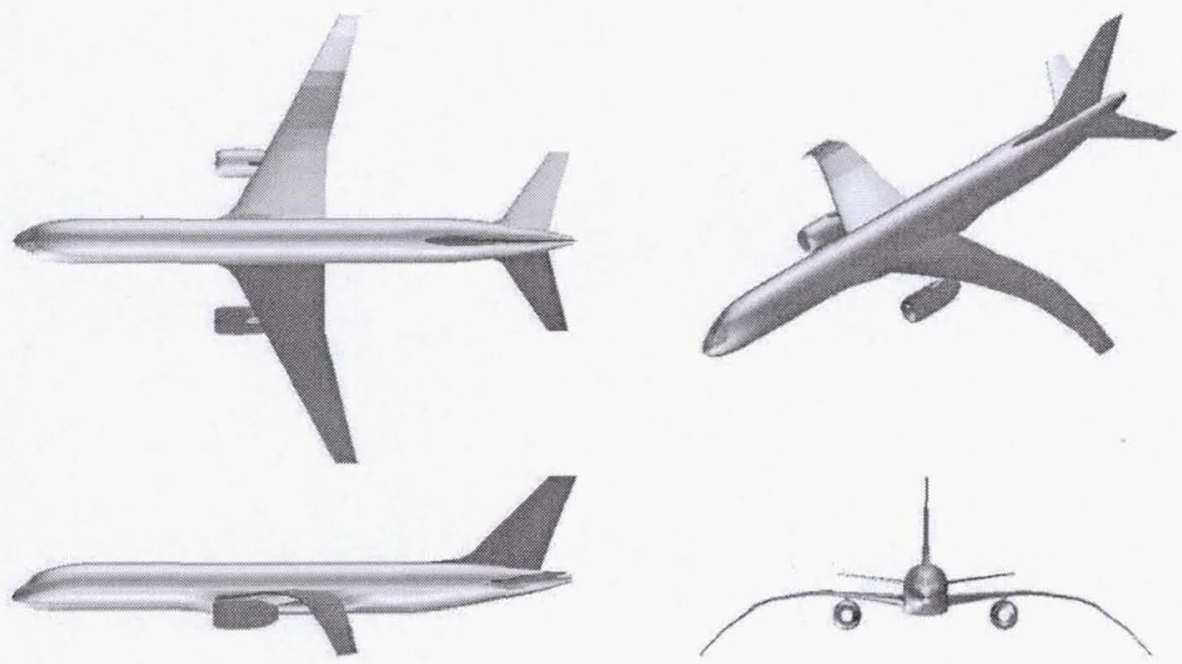

Figure 10 - Drooped-Wing Aircraft Concept

The optimal shape function parameters are $a_{4}=-7.5, a_{3}=-3.75, a_{2}=-8.75, b_{4}=-1.5, b_{3}=1.5$, and $b_{2}=-1.5$. Figures 11 and 12 show the mean line of the drooped wing shape and the wing wash-out twist distribution (positive nose-down). The wing tip has a very small ground clearance which can be problematic. Thus, the new wing design would have to incorporate a mechanism to articulate the wing tip portion to increase ground clearance. Nonetheless, notwithstanding practical implementation aspects of this concept, this optimal wing shape is quite interesting and revolutionary. The drooped wing shape in fact seems to mimic a seagull wing. Thus, it could be viewed as a biologically 
inspired concept. In fact, a literature search reveals that there was a study of a seagull-type wing in a wind tunnel at NASA Langley Research Center in 2006 [10]. The article stated that a seagull wing can be proved to have the greatest drag reduction - a 4 percent improvement over the theoretically best conventional aircraft wing.

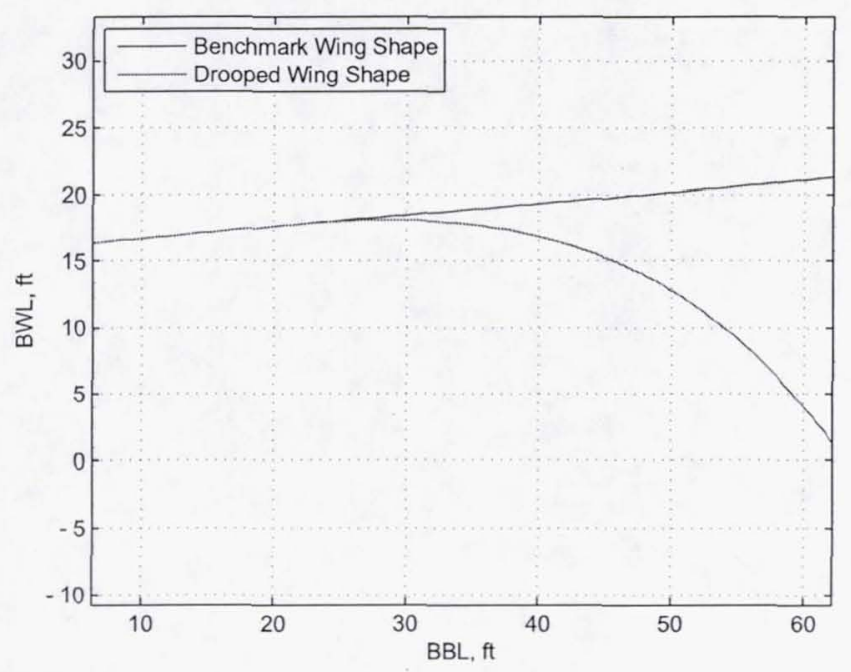

Figure 11 - Mean Line of Drooped Wing Shape

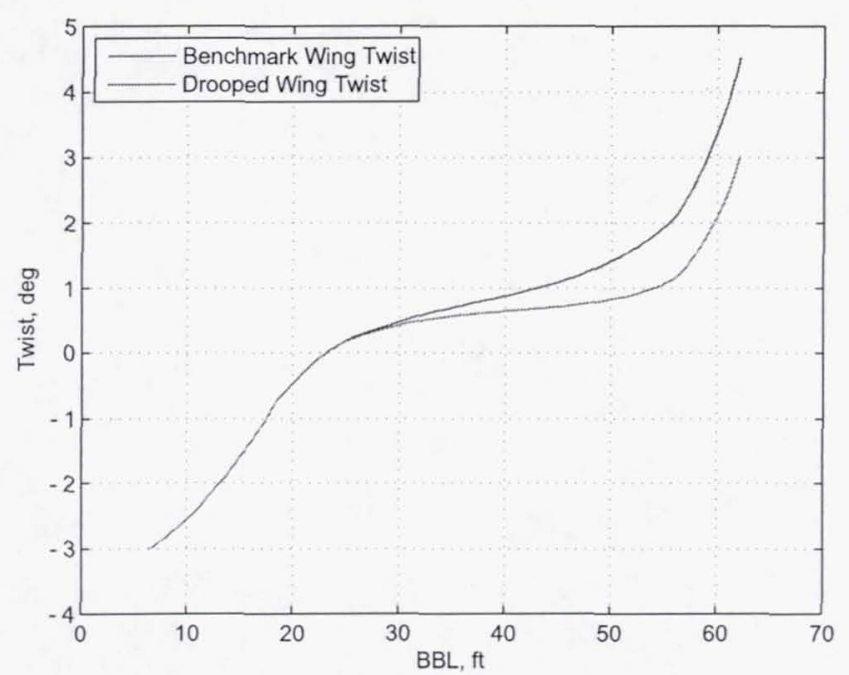

Figure 12 - Wash-Out Twist Distribution of Drooped Wing Shape

The low drag benefit of the drooped wing may be explained by aeroelasticity theory which shows that the local angle of attack of an elastic wing is a function of the wing curvature and twist according to [12, 13]

$$
\alpha_{c}=\frac{\alpha}{\cos \Lambda}-\gamma-W_{x} \tan \Lambda-\Theta
$$

where $\alpha_{c}$ is the section or local angle of attack, $\alpha$ is the aircraft angle of attack, $\Lambda$ is the wing sweep angle, $\gamma$ is the wing pre-twist wash-out angle (positive nose-down), $W_{x}$ is the wing flapwise bending deflection slope which represents the wing curvature, and $\Theta$ is the torsional deflection. 
Thus with a negative wing curvature, the local angle of attack increases. This increase causes the lift coefficient $C_{L}$ for the drooped wing to be higher than that for the straight wing of the baseline aircraft. Figure 13 is a plot of the lift coefficient $C_{L}$ versus $\alpha$, which shows that indeed the $C_{L}$ value for the drooped wing vehicle is larger than the $C_{L}$ value for the baseline aircraft at the same angle of attack. Thus, the computational result seems to corroborate the observation deduced from aeroelasticity theory. This would mean that for a given $C_{L}$ value at cruise, a drooped-wing aircraft would be trimmed at a lower angle of attack than the baseline aircraft. The reduction in the angle of attack results in the drag coefficient $C_{D}$ being moved closer to the minimum-drag angle of attack, which tends to be a small angle.

Another possible explanation for the potential drag reduction benefit of the drooped wing shape is that the negative curvature of the wing shape may effectively reduce the tendency for the high pressure flow field on the lower surface of the wing to form a flow circulation around the wing tip as a result of the low pressure region on the upper surface. Reducing tip circulation will directly result in a reduction in lift-induced drag.

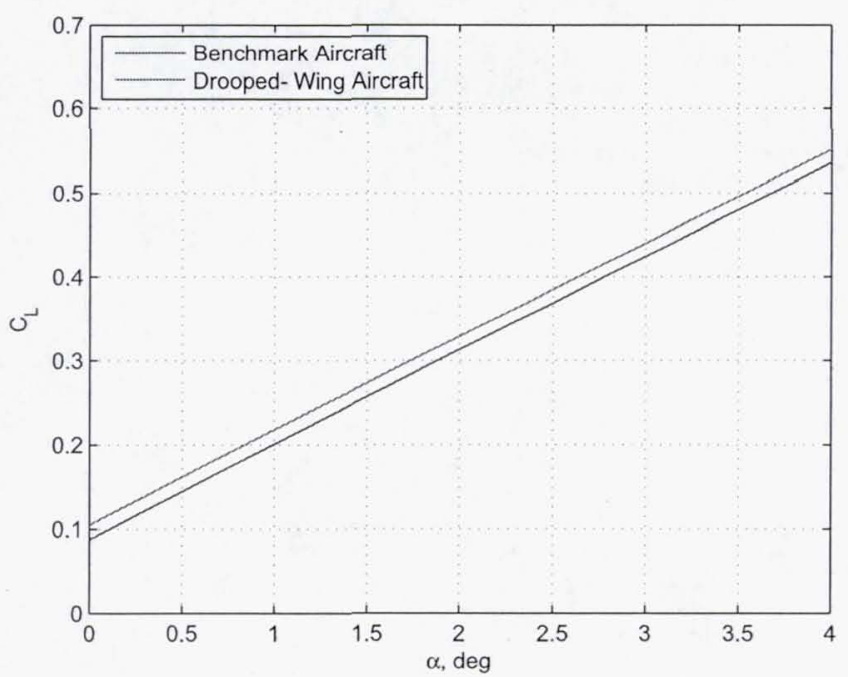

Figure $13-C_{L}$ vs. $\alpha$ for Drooped-Wing Aircraft

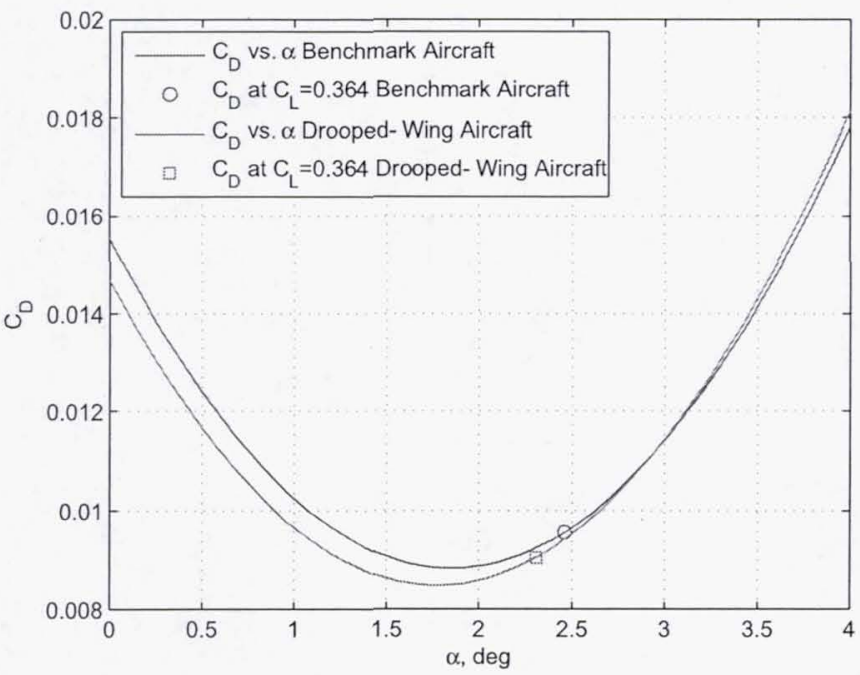

Figure 14 - $C_{D}$ vs. $\alpha$ for Drooped-Wing Aircraft 
Figures 14 and 15 show the drag coefficient as a function of the angle of attack and lift coefficient. As confirmed by the deduction, the $C_{D}$ value for the drooped-wing aircraft concept moves closer to the minimum-drag angle of attack, thereby resulting in a lower $C_{D}$ value than that for the baseline aircraft. The $C_{D}$ value for the drooped-wing aircraft is established by $C_{D}=0.00905$ as compared to $C_{D}=0.00956$ for the baseline aircraft. The overall induced drag reduction for the drooped-wing aircraft concept is 0.00051 or 5 drag counts. This represents a $5.3 \%$ reduction in the induced drag, which is considered significant.

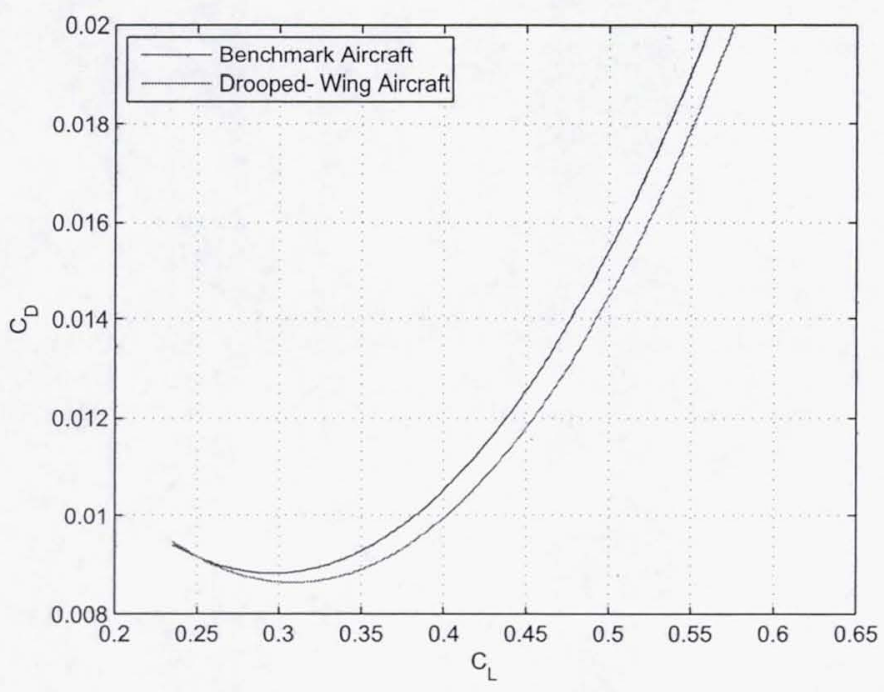

Figure 15 - Drag Polar for Drooped-Wing Aircraft

The sensitivity of the optimality of the drooped wing shape with the design cruise lift coefficient is also investigated. During cruise, as the fuel is burned, the aircraft weight is reduced and so is the cruise lift coefficient. For the worst case of empty fuel with $50,000 \mathrm{lbs}$ of fuel expanded, the cruise lift coefficient drops to $C_{L}=0.273$. An optimization cycle is performed for this design cruise lift coefficient. The results remain essentially unchanged with the same drooped wing shape being the most optimal. Thus, if the drooped-wing shape could be maintained at all times during cruise, a potential fuel saving could be realized. To maintain a constant wing shape in-flight would require an active wing shaping control system that would adjust the wing shape due to changes in the cruise condition. The active wing shape control will be further discussed.

Because the wing slope is much larger than the applied twist for the drooped-wing geometry, the effect of the wing droop tends to be more dominant than the effect of twist. For the same drooped wing shape, variations in the parameters $b_{i}, i=2,3,4$ appear to have little effect on the $C_{D}$ value for a small nose-up twist angle. For example, for the same drooped wing shape with $b_{4}=-1.5$ and $b_{3}=b_{2}=0$, the $C_{D}$ value is 0.00907 ; and with $b_{4}=b_{3}=b_{2}=0$, the $C_{D}$ value is 0.00912 . However, there are configurations with the same drooped wing shape that produce higher $C_{D}$. For example, with $b_{4}=b_{3}=b_{2}=-1.5$, the $C_{D}$ value is 0.00974 .

In addition, other drooped-wing aircraft concepts with lower wing tip deflections are also found to have lower $C_{D}$ values than that for the baseline aircraft. Figure 16 shows two other drooped-wing aircraft concepts and their minimum $C_{D}$ values for all the configurations with the same $a_{i}, i=2,3,4$.

Based on the observation above, it may be deduced that an aircraft with a negative wing curvature and proper wing twist may be aerodynamically more efficient than that with a straight horizontal wing. If this observation could be confirmed by wind tunnel testing, it could potentially be a new contribution to aeronautics. 

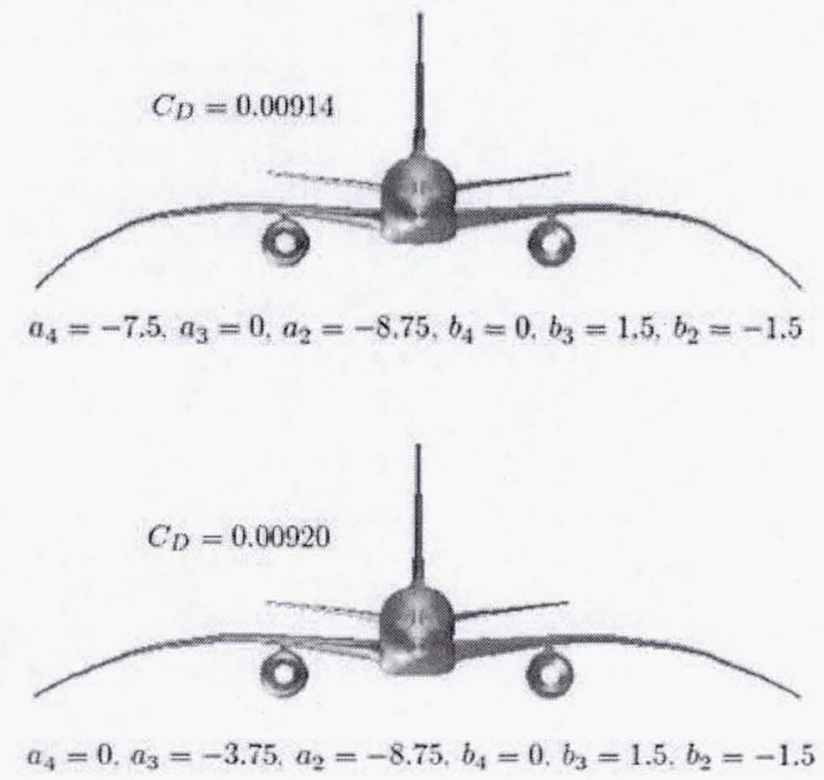

Figure 16 - Other Drooped-Wing Aircraft Concepts

\subsubsection{Inflected Wing Shape}

Since most of the low drag concepts are associated with drooped wing configurations, it is of interest in identifying a low drag concept with a positive wing curvature. The lowest drag with a positive wing tip deflection is found to be associated with a wing with a slight inflection in the mid-span. Thus, this concept is referred to as an inflected-wing aircraft as illustrated in Figure 17. The inflected wing shape is described by $a_{4}=7.5, a_{3}=3.75, a_{2}=-8.75, b_{4}=1.5$, $b_{3}=1.5$, and $b_{2}=-1.5$. Figures 18 and 19 show the mean line of the inflected wing shape and the wash-out twist distribution. The $C_{D}$ value for this concept is $C_{D}=0.00923$, which represents a 3.5\% reduction. Figure 20 shows the drag polar of the inflected-wing aircraft concept.
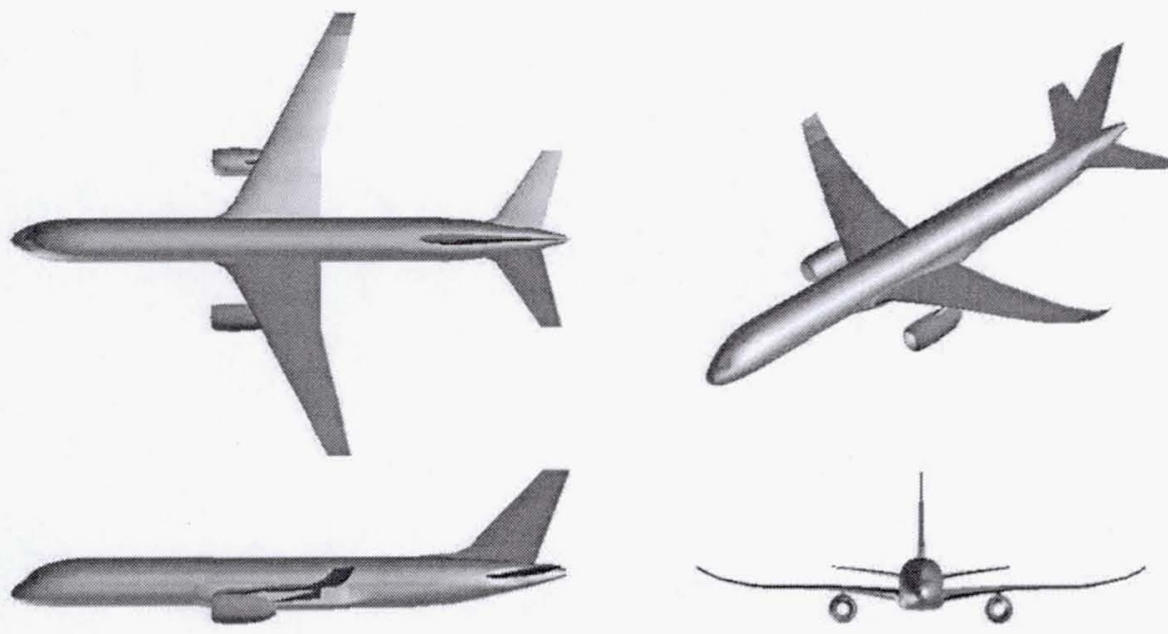

Figure 17 - Inflected-Wing Aircraft Concept 


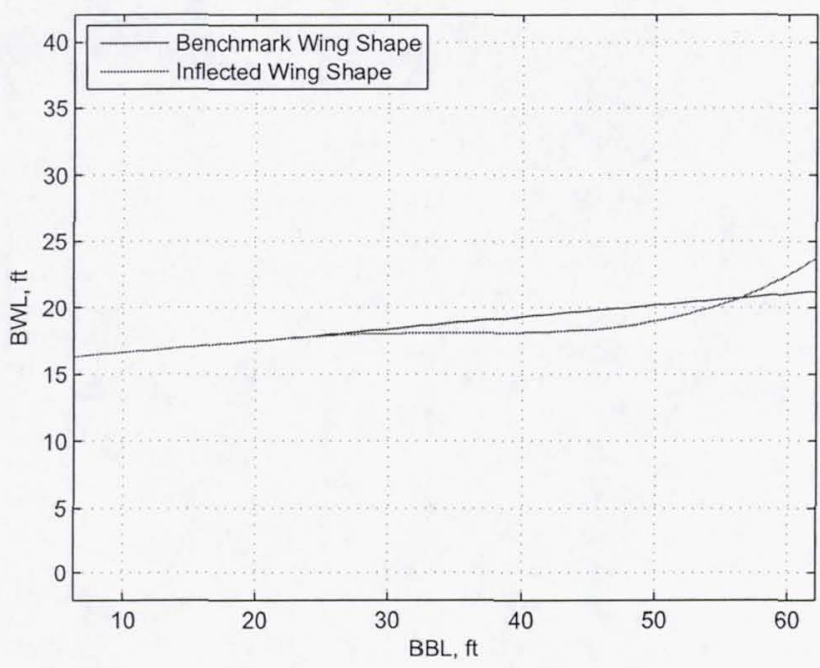

Figure 18 - Mean Line of Inflected Wing Shape

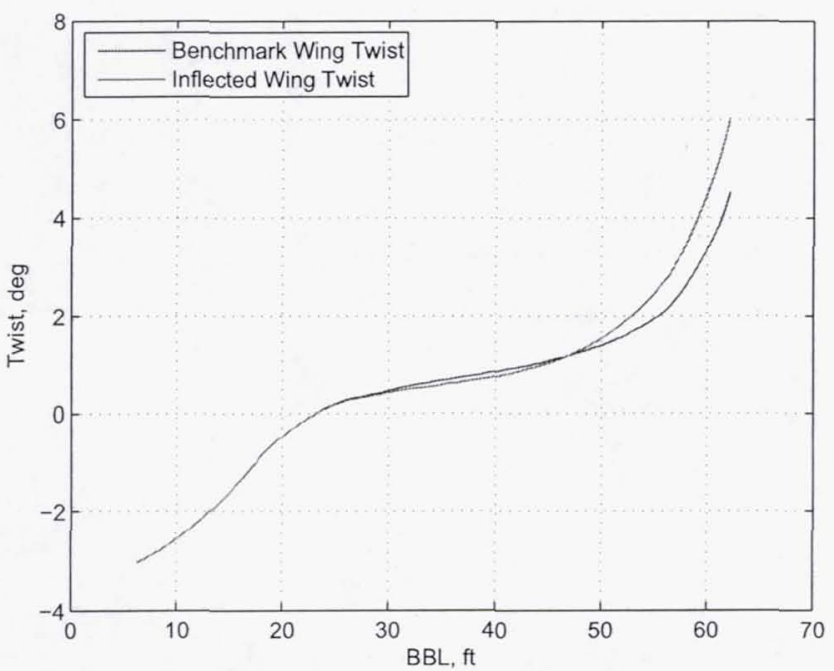

Figure 19 - Wash-Out Twist Distribution of Inflected Wing Shape 


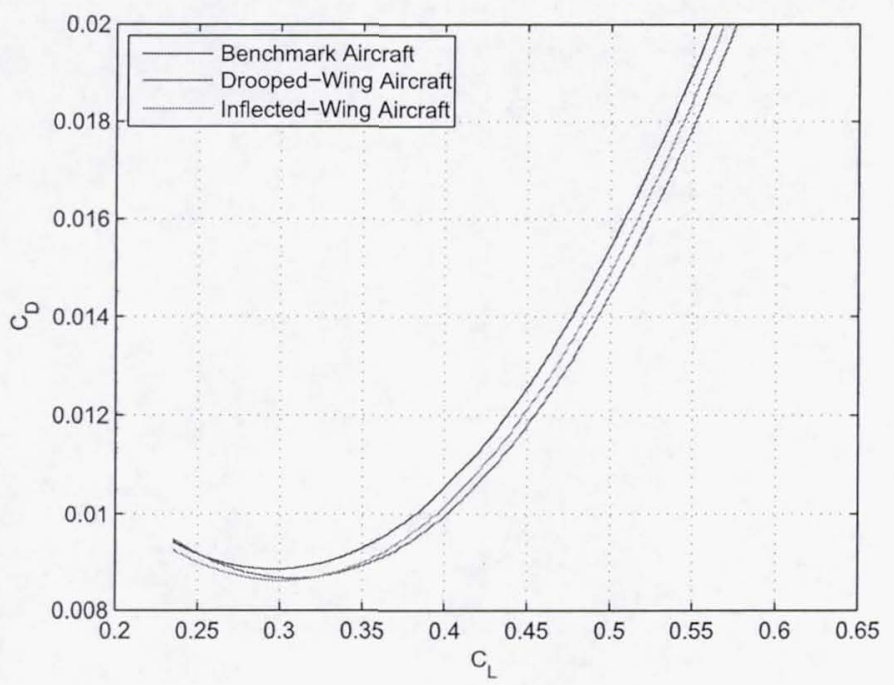

Figure 20 - Drag Polar for Inflected-Wing Aircraft

\subsection{Preliminary CFD Verification with Cart3D}

In order to check the potential induced drag benefits of some of the new concepts identified by the optimization, NASA's Cart3D simulation package was used in conjunction with adjoint-based mesh adaptation to verify the droopedwing and inflected-wing aircraft concepts. The adjoint-based mesh refinement module, used in this study, makes use of adjoint-weighted residual error-estimates to drive mesh adaptation in order to eradicate discretization error in the numerical solution ensuring accurate estimates of integrated forces such as lift or drag. For the current study, 12 mesh adaptation cycles were used. One complete mesh adapted flow solution took on average 7 hours on 64 CPUs of the Columbia supercomputer. Another valuable capability of Cart3D, which is used in the current study, is the ability to achieve a user-requested $C_{L}$ value by modifying the angle of attack at specified time steps. This capability is helpful in comparing induced-drag benefits across different geometries. A $C_{L}$ value of 0.6 is chosen for the current study as this results in the best convergence of drag. For a lower $C_{L}$ value such as the design cruise $C_{L}$ value of 0.364 , there could be an issue with a solution convergence.

\subsubsection{Geometry and Mesh Generation}

The geometry used for this study is a scaled-up version of a $5.5 \%$ model of the GTM obtained from NASA Langley. A ProE assembly of all the parts was provided which was then converted to a water-tight CFD quality triangulation by triangulating each part individually and then intersecting. This is the same baseline triangulation used in previous work [5]. Once this water-tight geometry has been created, Cart3D automatically generates an initial Cartesian volume mesh around the triangulation. Cart3D then refines this initial coarse mesh in a manner described in the next section. A user-prescribed input in Cart3D is the aspect ratio of the volume cells. With the flow primarily along the chordwise direction of the wing, there is no need for the same resolution in the spanwise direction. Hence, present computations use a cell aspect ratio of about two in the spanwise direction.

\subsubsection{Wing Bending Implementation}

In order to properly model the deformation of the baseline aircraft wings, a program was written to manipulate the triangulation. This program reads in the baseline aircraft configuration, applies a given twist angle about a precomputed centroidal axis for the wings prescribed by the twist shape function in Eq. (2.10), deflects the wing in the normal direction according to the bending shape function prescribed by Eq. (2.11), and then outputs a final water-tight, CFD quality triangulation. Both twist and bending deflection are applied outboard of the engine pylons on both wings. The exact location is at a spanwise location of $22.12 \mathrm{ft}$ (BBL 22.12 ft) from the aircraft $x$ - axis. The location between 
this engine pylon location and the wing tip is normalized such that $u=0$ at the pylon and $u=1$ at the wing tip, where $u=\left(y_{V}-y_{t}\right) /\left(y_{t}-y_{e}\right)$ is the normalized coordinate along the wing span. This can be seen in Figure 20.

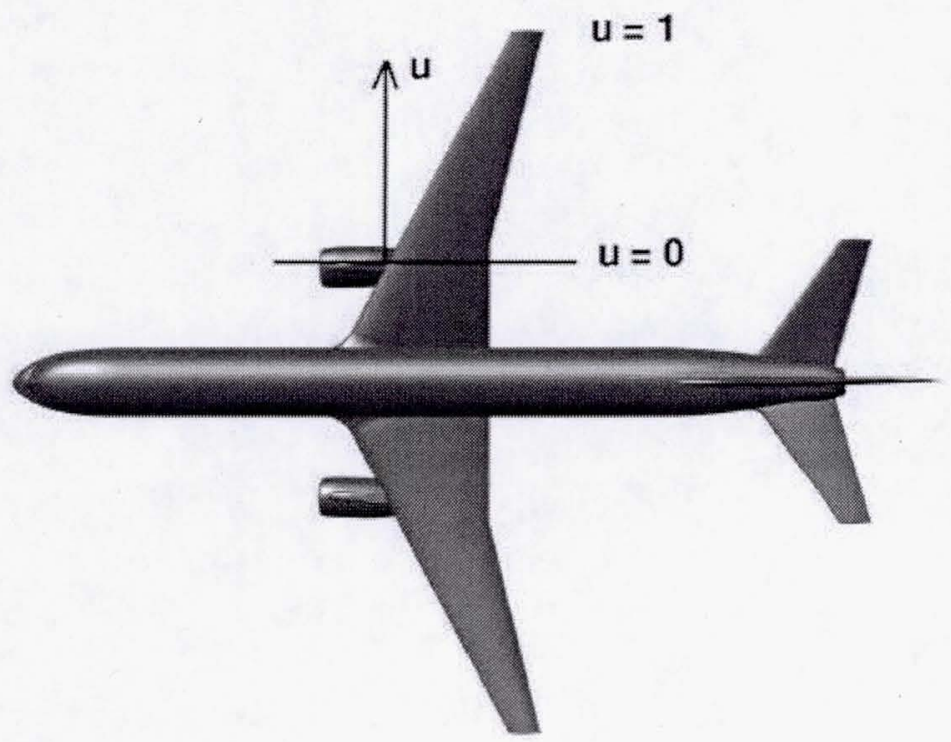

Figure 21 - Shape Function Schematic

The deformed geometries (under the prescribed bending deflection, no twist) are shown in Figure 22. The drooped wing and inflected wing geometries were found to be optimal in terms of induced-drag reduction using VORVIEW. All of the cases for the current study were examined with bending deflection only. Future studies will include both bending deflection and twist.
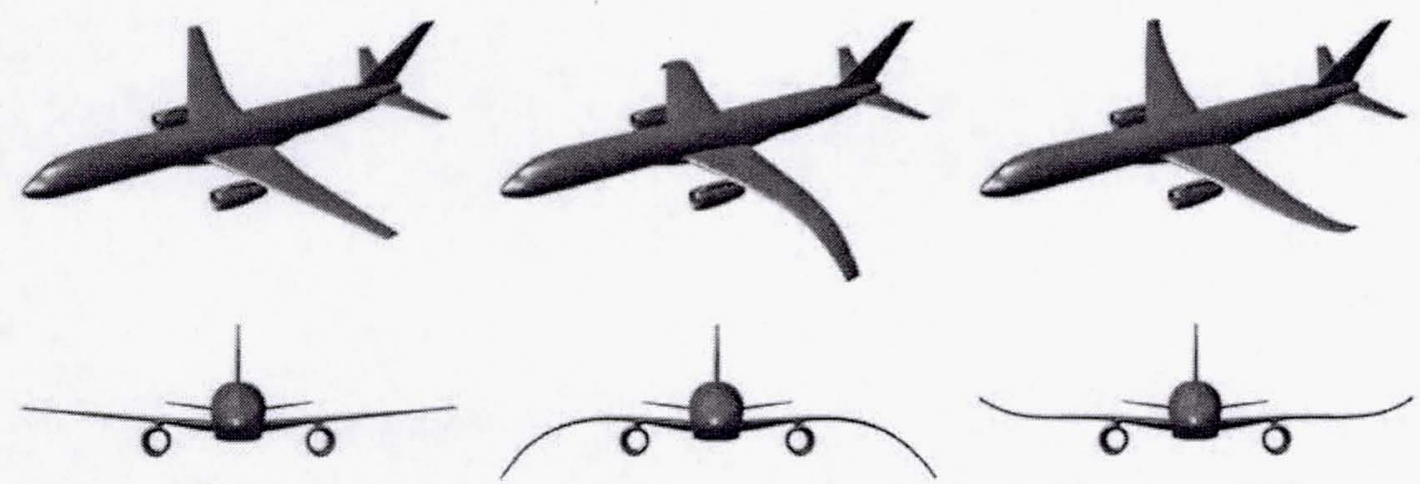

(a) Benchmark

(b) Drooped Wing

$$
\begin{gathered}
a_{4}=-3.5, a_{3}=-3.75, a_{2}=-8.75 \\
b_{4}=b_{3}=b_{2}=0
\end{gathered}
$$

(c) Inflected Wing

$$
a_{4}=3.5, a_{3}=3.75, a_{2}=-8.75
$$$$
b_{4}=b_{3}=b_{2}=0
$$

Figure 22 - Deformed Geometries under Prescribed Bending Deflection (no Twist)

The functional used to drive mesh adaptation is given by

$$
J=0.01 C_{L}+C_{D}
$$

Since the main goal of the study is to capture the induced drag of the wings, and because the $C_{L}$ value is much greater than the $C_{D}$ value, therefore converges faster than drag, a heavier weighting is given to the drag coefficient. 


\subsubsection{Results}

The following cases were run at a Mach number of 0.3 for verification with VORVIEW. Figures 23 and 24 display Mach contours and surface pressures of the three aircraft configurations. The Mach number near the wing tip of the drooped-wing aircraft appears lower than the Mach number for both the baseline aircraft and inflected-wing aircraft which seem to have a similar Mach number distribution. The pressure distribution over the upper surface of the wings for the three configurations appears to be quite similar, although there is a stronger low pressure region at the wing leading edge in the baseline aircraft. This could translate into a faster localized flow field in the leading edge region that might result in shock-induced separation at higher Mach number. Figure 25 is the convergence plot for the functional and error convergence.

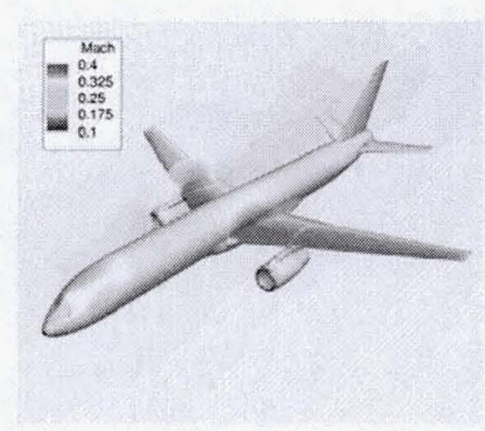

(a) Benchmark

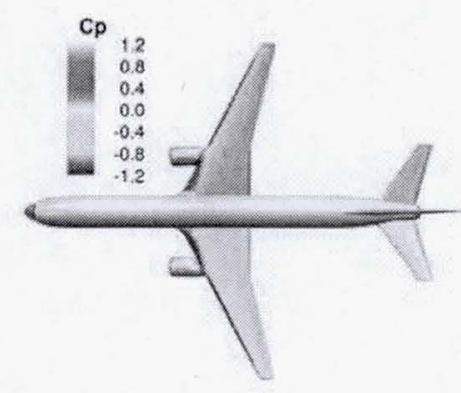

(a) Benchmark

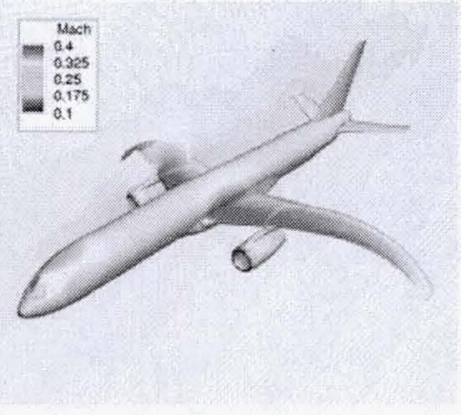

(b) Drooped Wing

Figure 23 - Mach Contours

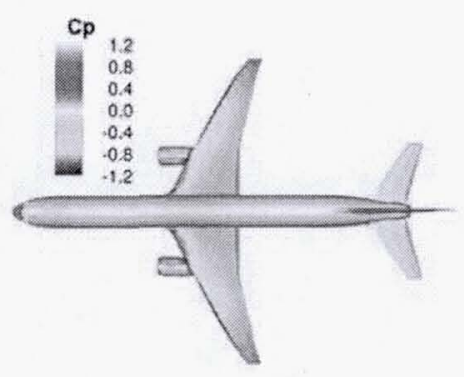

(b) Drooped Wing

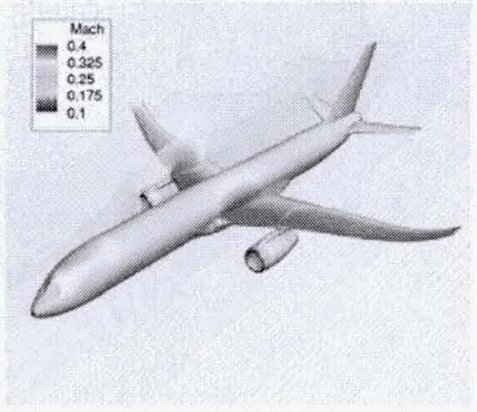

(c) Inflected Wing

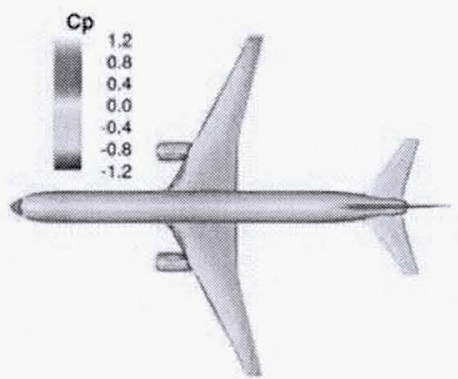

(c) Inflected Wing

Figure 24 - Surface $c_{p}$ Contours

Table 3 below summarizes the Cart3D results for the three aircraft configurations. The parameter $e$ in the table represents the span efficiency factor for the wings, where $e=C_{L}^{2} /\left(\pi A R C_{D}\right)$ is 1 for the ideal elliptical lift distribution. Based on these results, it appears that the inflected-wing aircraft configuration provides a better benefit in terms of induced-drag reduction. The inflected-wing configuration yields a $2.3 \%$ improvement over the baseline aircraft configuration in span efficiency and a $2.0 \%$ reduction in drag. The drooped-wing aircraft configuration, however, does not appear to provide any improvement. This could be due to not having the prescribed twists applied in the geometries since the twist can potentially affect the local angle of attack on a wing section.

\begin{tabular}{|c||c|c|c|c|}
\hline & $\alpha, \mathrm{deg}$ & $C_{L}$ & $C_{D}$ & $e$ \\
\hline \hline Baseline & 4.94 & 0.6 & 0.0224 & 0.731 \\
\hline Drooped Wing & 5.15 & 0.6 & 0.0224 & 0.733 \\
\hline Inflected Wing & 4.92 & 0.6 & 0.0220 & 0.748 \\
\hline
\end{tabular}

Table 3 - Drag Reduction at Mach 0.3 
For comparison, VORVIEW results for $C_{L}=0.6$ for the drooped-wing and inflected-wing aircraft configurations are computed. The results are from the optimal configurations with twists incorporated, so they might not give a direct comparison. Table 4 shows the drag comparison among the three aircraft configurations. The relative benefits of the induced drag reduction for the drooped-wing and inflected-wing aircraft configurations remain about the same as the optimization results.

\begin{tabular}{|c||c|c|c|c|}
\hline & $\alpha, \mathrm{deg}$ & $C_{L}$ & $C_{D}$ & $e$ \\
\hline \hline Baseline & 4.50 & 0.6 & 0.0235 & 0.696 \\
\hline Drooped Wing & 4.45 & 0.6 & 0.0224 & 0.731 \\
\hline Inflected Wing & 4.51 & 0.6 & 0.0229 & 0.716 \\
\hline
\end{tabular}

Table 4 - Drag Reduction at Mach 0.8

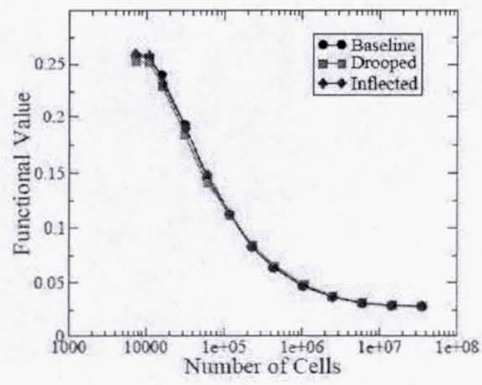

(a) Funetional Convergence

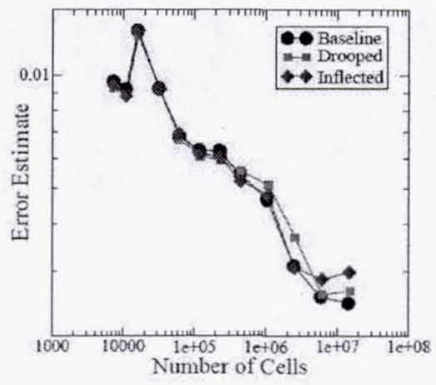

(b) Error Convergence

Figure 25 - Functional and Error Convergence

In summary, the inflected wing geometry shows a beneficial effect; however, potential does exist for the drooped wing case as well. If the wing tip bending deflection was incorporated with wing twist so that the same lift could be achieved at a lower angle of attack, the drooped wing geometry could provide a larger benefit. In any case, no definitive conclusion could be made in regards to the merits of the drooped-wing and inflected-wing aircraft configurations at this point since the validation effort is very limited in scope due to the time and resource constraints. Given that these concepts could be potentially beneficial for induced drag reduction, a further follow-on effort should be considered to conduct a more thorough analysis that includes the effect of the optimal wing twists which are neglected in this study.

\section{Elastic Wing Shaping Control}

Based on the optimization results, the drooped-wing aircraft concept is selected for the active wing shaping control design since it provides the best induced drag reduction. Since the optimal wing shape is not sensitive to changes in the cruise lift coefficient, to maintain the best cruise efficiency, the wing shape would need to be actively controlled. A concept of operation is now defined as follows:

- The design point for wing shaping control actuator requirements is defined to be at the half way point of cruise at $30,000 \mathrm{ft}$ corresponding to an aircraft weight of $175,000 \mathrm{lbs}$ with $50 \%$ fuel in the tank.

- The active wing shaping control actuator will be a series of leading edge slats and trailing edge flaps.

- The wing shape at the design point corresponds to the optimal drooped-wing shape. This would mean that the as-built wing shape would be the optimal drooped wing shape minus the differential bending and torsional deflections at $1-\mathrm{g}$ loading at the design point. Under the 1-g loading at cruise, the wing shape is aerodynamically loaded and deflected into the optimal drooped-wing shape with no flap or slat deflection.

- At the start of cruise, the aircraft weight is $190,000 \mathrm{lbs}$ with $80 \%$ fuel in the tank. This would correspond to a higher wing loading that causes the wing shape to move up from the optimal drooped wing shape. Active wing shaping control flaps and slats are deployed to bring the wing shape back to the optimal drooped wing shape. This would result in a lower wing loading that would cause the aircraft to decrease in altitude. 
- At the end of cruise, the aircraft weight is $160,000 \mathrm{lbs}$ with $20 \%$ fuel in the tank. This would correspond to a lower wing loading that causes the wing shape to move down from the optimal drooped wing shape. Active wing shaping control flaps and slats are deployed to bring the wing shape back to the optimal drooped wing shape. This would result in a higher wing loading that would cause the aircraft to increase in altitude.

A novel wing shaping control actuator concept is developed in this study; namely, a distributed variable camber continuous trailing edge flap system.

\subsection{Low-Drag Variable Camber Continuous Trailing Edge Flap Concept}

In a conventional flap design, individual flaps are actuated independently. As a result, the trailing edge of a wing formed by the flap deflections is discontinuous. This discontinuity is a source of drag penalty as well as acoustic emissions. The VORVIEW results show that the drag penalty due to the conventional flap system is substantial. One way to reduce the drag penalty is to use a single flap surface over a wide wing span. However, this would compromise the flexibility and effectiveness of wing shaping control. A novel new flap concept is thus introduced to address the drag reduction goal of the study. This flap concept is called a variable camber continuous trailing edge flap. Figure 26 illustrates the continuous flap concept.

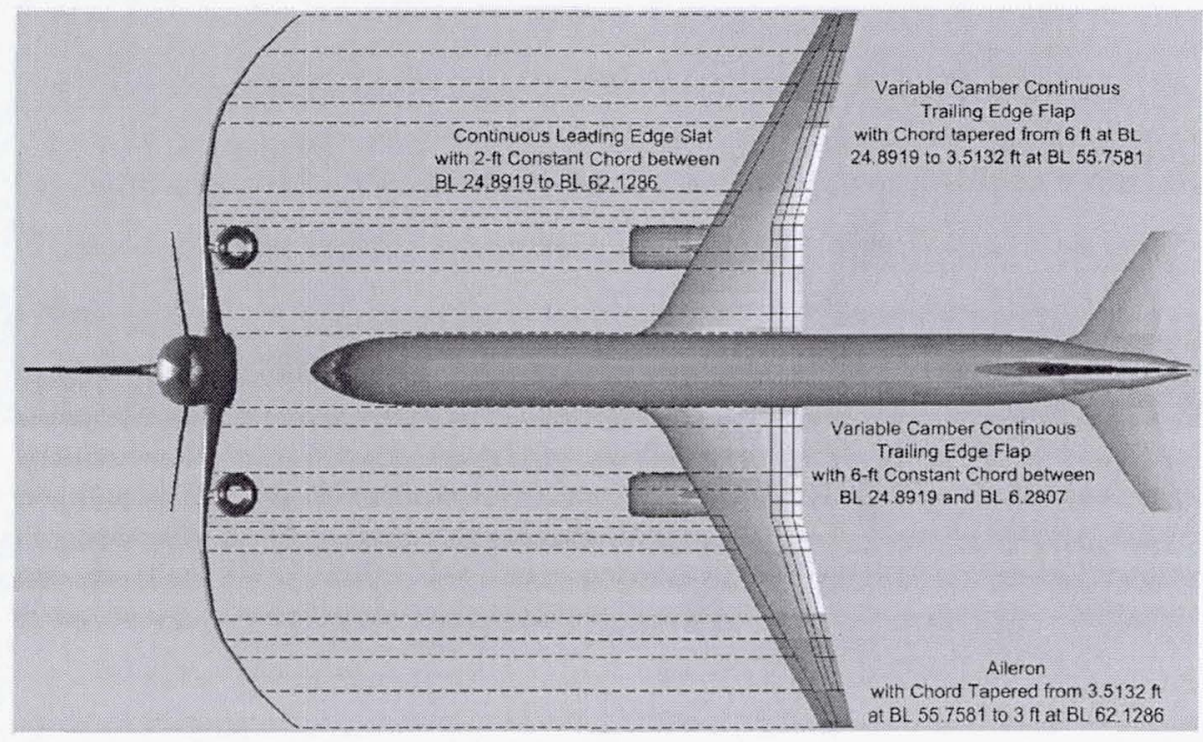

Figure 26 - Variable Camber Continuous Trailing Edge Flap System

The two main features of this flap concepts that provide significant drag reduction benefits are:

- Variable camber flap - The flap chord is comprised of three chordwise segments of equal chord length as shown in Figure 27. These three flap segments are actuated in unison when a flap deflection command is given. Each flap segment is deflected by an angle equal to one third of the commanded flap deflection relative to each other. For example, for a commanded flap deflection of $12^{\circ}$, flap segment 1 which is positioned next to the wing is deflected $4^{\circ}$, flap segment 2 that follows flap segment 1 is deflected $8^{\circ}$, and flap segment 3 at the trailing edge is deflected by $12^{\circ}$. Thus

$$
\begin{gathered}
f_{1}=\frac{f_{c}}{3} \\
f_{2}=\frac{2 f_{c}}{3} \\
f_{3}=f_{c}
\end{gathered}
$$

where $f_{c}$ is the commanded flap deflection. 
The camber angle of the flap is the difference between between $f_{3}$ and $f_{1}$. Thus, the variable camber angle $\chi=2 f_{c} / 3$ is a function of the commanded flap deflection. A cambered flap is more effective in producing lift than a straight uncambered flap.

The variable camber flap produces about the same downwash as a simple plain flap deflected by the same angle, as seen in Figure 26. However, the normal surface area of the variable camber flap exposed to the flow field is significantly reduced. Thus, the drag reduction benefit of the variable camber flap is realized since the pressure drag across the flap surface is reduced due to less exposed normal surface area.

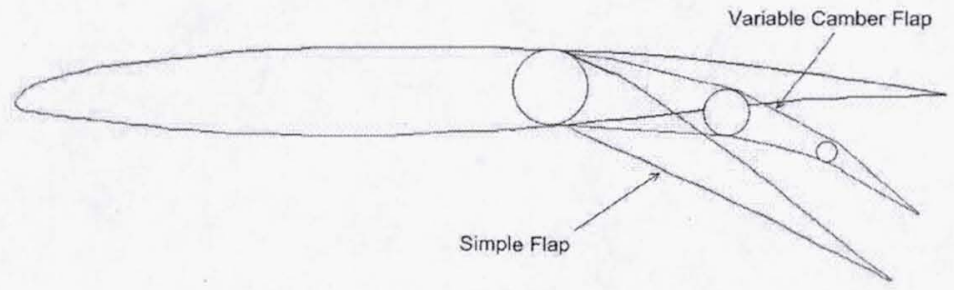

Figure 27 -Variable Camber Flap

- Continuous trailing edge flap - The continuous flap is comprised of 12 spanwise segments to form a continuous trailing edge when the flap is deflected. This continuous trailing edge would eliminate vortices which otherwise would have formed at the conventional flap discontinuity in the trailing edge region. By reducing or eliminating vortex formation, drag losses as well as acoustic emissions from turbulence could be attenuated. Thus, this feature further provides a drag reduction benefit in addition to the variable camber flap concept.

The flap spans the wing trailing edge from BBL $6.2807 \mathrm{ft}$, which is abutted to the fuselage, to BBL $55.7581 \mathrm{ft}$. The flap deflection angle varies continuously from zero at these two BBL stations to the maximum commanded flap deflection at BBL $24.8919 \mathrm{ft}$. The chord is tapered from $6 \mathrm{ft}$ at BBL $24.8919 \mathrm{ft}$ to $3.5132 \mathrm{ft}$ at BBL 55.7581 $\mathrm{ft}$, and then remains constant at $6 \mathrm{ft}$ between BBL $6.2807 \mathrm{ft}$ to BBL $24.8919 \mathrm{ft}$. A theoretical smooth trailing edge shape is generated by a 5 th-degree polynomial to enforce the boundary conditions as follows:

$$
f_{n}(y)=a_{5} y^{5}+a_{4} y^{4}+a_{3} y^{3}+a_{2} y^{2}+a_{1} y+a_{0}
$$

where $f_{n}$ is the continuous flap deflection of flap $n, n=1,2,3, y$ is the BBL station, and $a_{i}, i=1,2,3,4,5$ are the polynomial coefficients that satisfy the following boundary conditions

$$
\begin{gathered}
f_{n}\left(y_{1}=6.2807\right)=0 \\
f_{n}^{\prime}\left(y_{1}=6.2807\right)=0 \\
f_{n}\left(y_{2}=24.8919\right)=\frac{n f_{c}}{3} \\
f_{n}^{\prime}\left(y_{2}=24.8919\right)=0 \\
f_{n}\left(y_{3}=55.7581\right)=0 \\
f_{n}^{\prime}\left(y_{3}=55.7581\right)=0
\end{gathered}
$$

The coefficients $a_{i}, i=1,2,3,4,5$ are determined as

$$
\left[\begin{array}{l}
a_{5} \\
a_{4} \\
a_{3} \\
a_{2} \\
a_{1} \\
a_{0}
\end{array}\right]=\left[\begin{array}{cccccc}
y_{1}^{5} & y_{1}^{4} & y_{1}^{3} & y_{1}^{2} & y_{1} & 1 \\
5 y_{1}^{4} & 4 y_{1}^{3} & 3 y_{1}^{2} & 2 y_{1} & 1 & 0 \\
y_{2}^{5} & y_{2}^{4} & y_{2}^{3} & y_{2}^{2} & y_{2} & 1 \\
5 y_{2}^{4} & 4 y^{3} & 3 y_{2}^{2} & 2 y_{2} & 1 & 0 \\
y_{3}^{5} & y_{3}^{4} & y_{3}^{3} & y_{3}^{2} & y_{3} & 1 \\
5 y_{3}^{4} & 4 y_{3}^{3} & 3 y_{3}^{2} & 2 y_{3} & 1 & 0
\end{array}\right]^{-1}\left[\begin{array}{c}
0 \\
0 \\
\frac{n f_{c}}{3} \\
0 \\
0 \\
0
\end{array}\right]=n f_{c}\left[\begin{array}{c}
-1.2929 \times 10^{-7} \\
2.2291 \times 10^{-5} \\
-4.5450 \times 10^{-4} \\
3.4045 \times 10^{-2} \\
-2.8738 \times 10^{-1} \\
7.6635 \times 10^{-1}
\end{array}\right]
$$

Figure 28 shows the theoretical continuous trailing edge flap deflection curve and the flap chord distribution. 

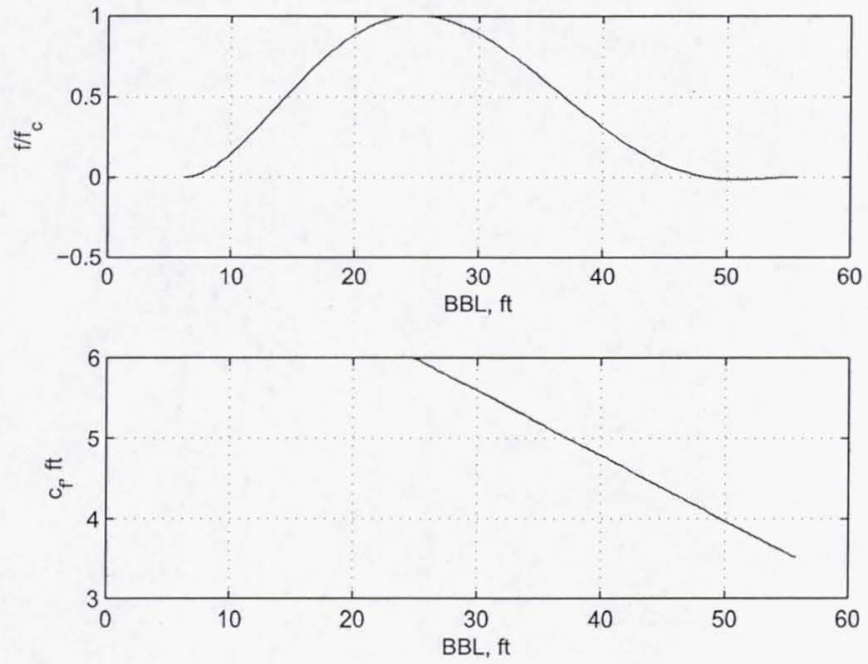

Figure 28 - Theoretical Continuous Trailing Edge Flap Deflection

To approximate this continuous trailing edge curve, the continuous flap system can be made of 12 continuous flap segments where they join together to form a piecewise continuous trailing edge. Each flap segment is designed to be actuated in relation to the adjacent flap segments in the spanwise direction to form a continuous trailing edge, as well as in the chordwise direction to form a desired camber. Tables 5 and 6 show the BBL stations of the 12 flap segments numbered from 1 to 12 from outboard to inboard.

\begin{tabular}{|c|c|c|c|c|c|c|}
\hline & 1 & 2 & 3 & 4 & 5 & 6 \\
\hline \hline Outboard BBL & 55.7581 & 49.1475 & 46.2442 & 42.6498 & 31.0369 & 28.4101 \\
\hline Inboard BBL & 49.1475 & 46.2442 & 42.6498 & 31.0369 & 28.4101 & 26.7512 \\
\hline Outboard $3 f_{n} / n f_{c}$ & 0 & -0.0085 & 0.0390 & 0.1669 & 0.8374 & 0.9433 \\
\hline Inboard $3 f_{n} / n f_{c}$ & -0.0085 & 0.0390 & 0.1669 & 0.8374 & 0.9433 & 0.9837 \\
\hline
\end{tabular}

Table 5 - Continuous Trailing Edge Flap Segments 1 to 6

\begin{tabular}{|c|c|c|c|c|c|c|}
\hline & 7 & 8 & 9 & 10 & 11 & 12 \\
\hline \hline Outboard BBL & 26.7512 & 24.8919 & 22.7419 & 20.6682 & 17.6267 & 9.4700 \\
\hline Inboard BBL & 24.8919 & 22.7419 & 20.6682 & 17.6267 & 9.4700 & 6.2807 \\
\hline Outboard $3 f_{n} / n f_{c}$ & 0.9837 & 1.0000 & 0.9772 & 0.9115 & 0.7430 & 0.1095 \\
\hline Inboard $3 f_{n} / n f_{c}$ & 1.0000 & 0.9772 & 0.9115 & 0.7430 & 0.1095 & 0 \\
\hline
\end{tabular}

Table 6 - Continuous Trailing Edge Flap Segments 7 to 12

Figure 29 shows the BBL stations of the 12 flap segments. 


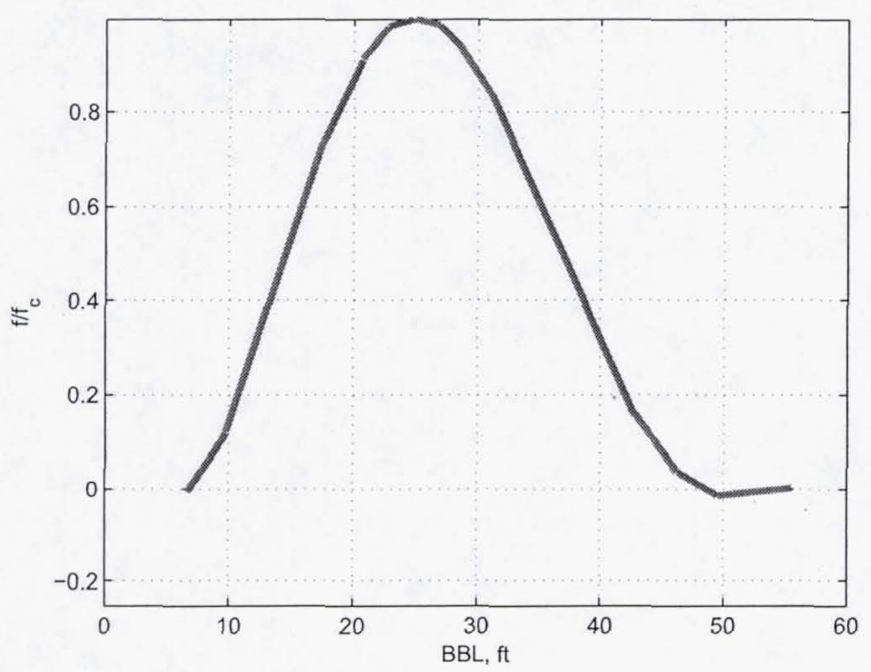

Figure 29 - Continuous Trailing Edge Flap Segments

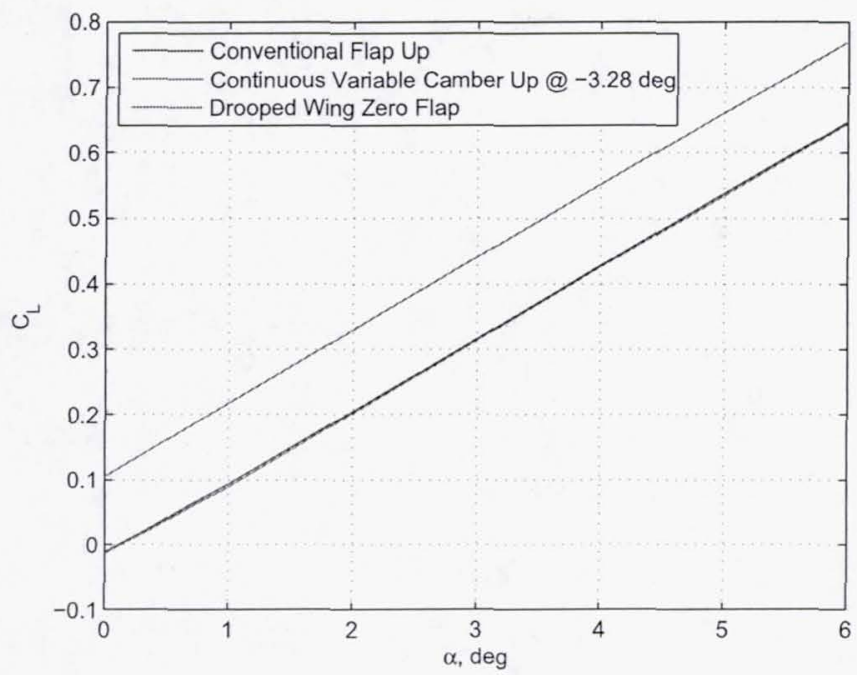

Figure $30-C_{L}$ versus $\alpha$ for Negative Flaps at Start of Cruise

The variable camber continuous trailing edge flap concept is implemented in VORVIEW to estimate the potential drag reduction benefit. The commanded flap deflection is adjusted until the $C_{L}$ versus $\alpha$ curve matches that for the drooped-wing aircraft with a conventional flap at the start and end of cruise. Figures 30 and 31 show the $C_{L}$ versus $\alpha$ curves for the flap deflections at the start and end of cruise. The drag reduction benefits of the variable camber continuous trailing flap system can be demonstrated by Figures 32 and 33 . It is apparent that the variable camber continuous trailing edge flap concept offers substantial drag reduction benefits. The incremental $C_{D}$ values are 0.00216 at the start and 0.00234 at the end of cruise. The results show drag reduction benefits ranging from $66 \%$ at the start of cruise to $46 \%$ at the end of cruise. Moreover, it is noted that the $C_{D}$ values for the variable camber continuous trailing edge flap are even lower than the baseline values for the drooped wing for $C_{L}$ values greater than 0.43 . This implies that the variable camber continuous flap concept is more aerodynamically efficient in producing high lift. 


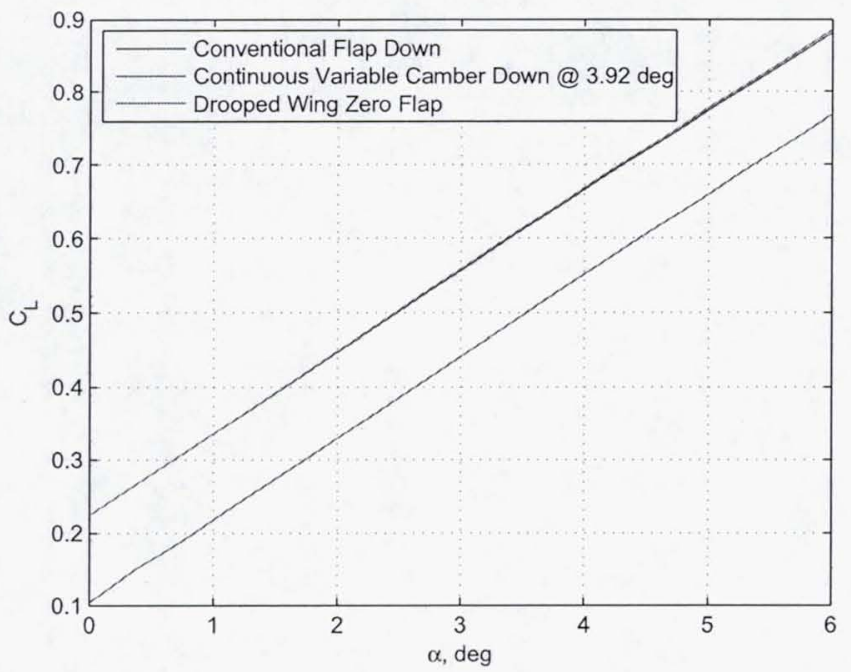

Figure $31-C_{L}$ versus $\alpha$ for Positive Flaps at End of Cruise

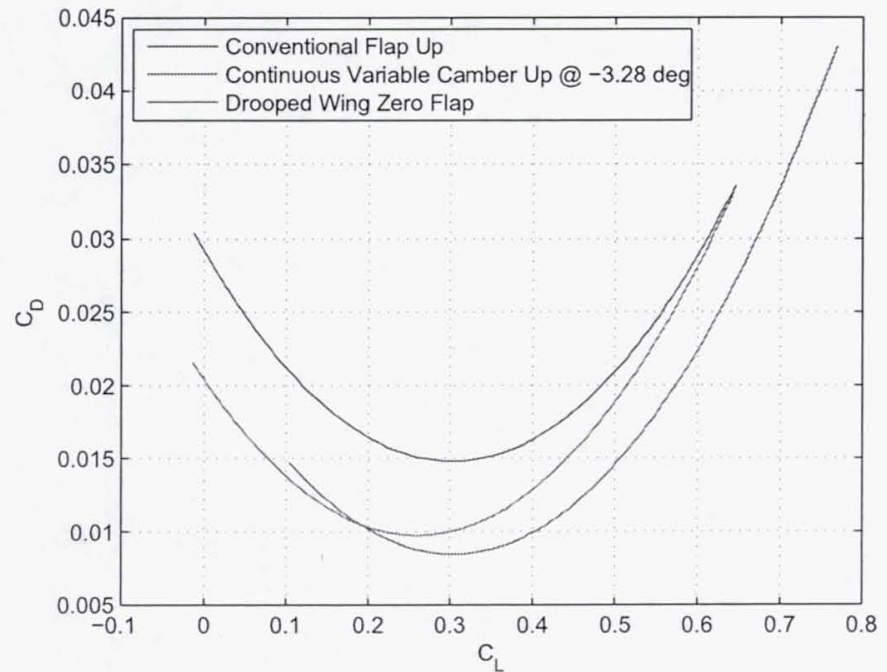

Figure 32 - Drag Polars for Plain Discrete Flaps and Variable Camber Continuous T.E. Flap at Negative Deflection 


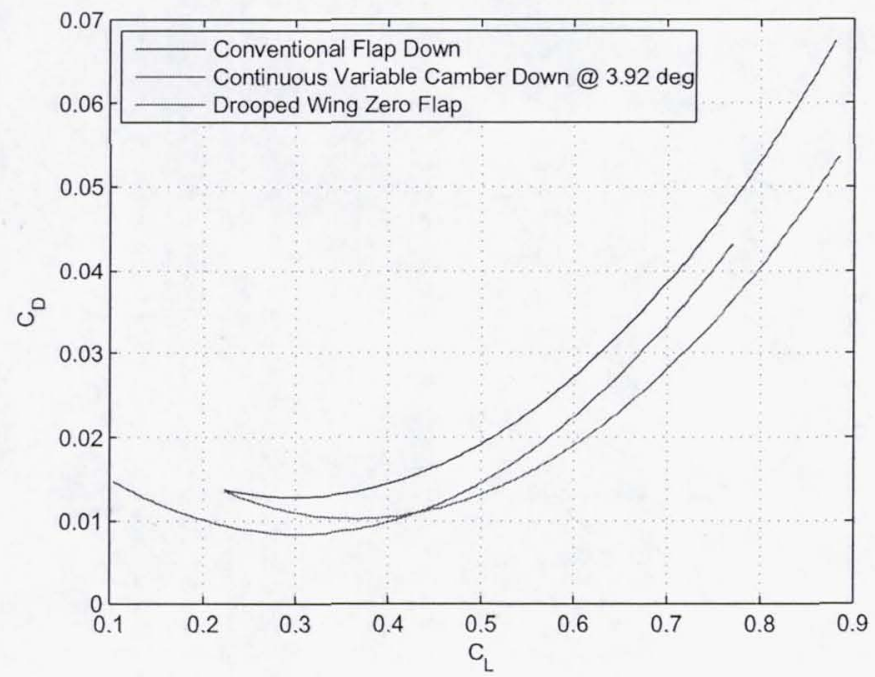

Figure 33 - Drag Polars for Plain Discrete Flaps and Variable Camber Continuous T.E. Flap at Positive Deflection

\section{Performance Analysis}

The ultimate goal of optimal wing shape and wing shaping control is to reduce fuel burn. The drooped-wing shape aircraft configuration is chosen for comparison with the baseline aircraft.

\subsection{Aeroelastic Deflection Effect}

Aeroelastic deflection can affect aircraft aerodynamics. As the aircraft cruises, fuel is burned and the wing loading is reduced, thereby causing the wing shape to displace downward and the wing twist to pitch nose-down. To assess the relative benefit of wing shaping control, a static aeroelastic deflection analysis is conducted to compute the wing bending and torsional deflections. The results are as shown in Figures 34 and 35.
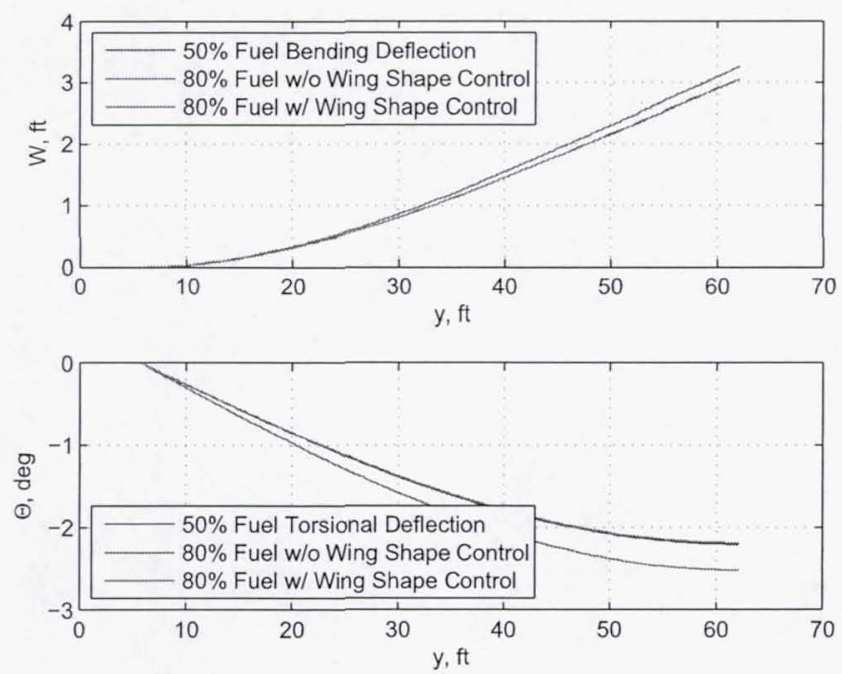

Figure 34 - Wing Deflections at Start of Cruise 

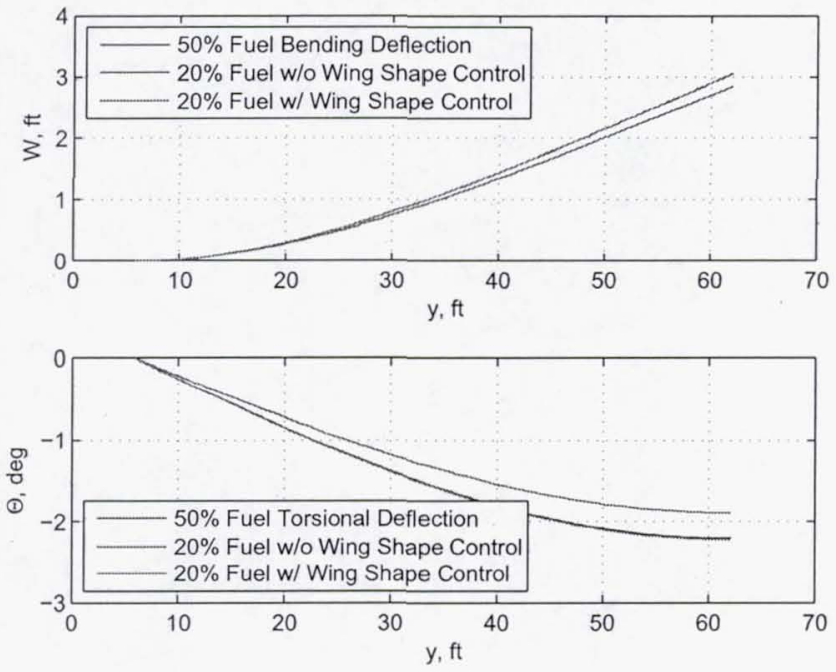

Figure 35 - Wing Deflections at End of Cruise

The change in wing shape can cause a drag penalty since the wing shape no longer retains its optimal droopedwing shape. The objective of wing shaping control is to restore the wing shape back to its optimal shape through flap and slat deployments. However, the drag penalty due to flap and slat deflections can negate any benefit attained from wing shaping control. If the optimal wing shape occurs at the half-way point in cruise, then the wing deflections will be due to the $\pm 30 \%$ fuel weight variations between the start and end of cruise. The wing bending and torsion deflection shapes are then superimposed on top of the drooped-wing shape to create a new wing shape for which new aerodynamic coefficients are computed using VORVIEW.

The results do indicate a significant drag penalty as the wing shape moves away its optimal shape. Figure 36 shows the drag polars at the three points in the cruise envelope: $80 \%$ fuel, $50 \%$ fuel, and $20 \%$ fuel.

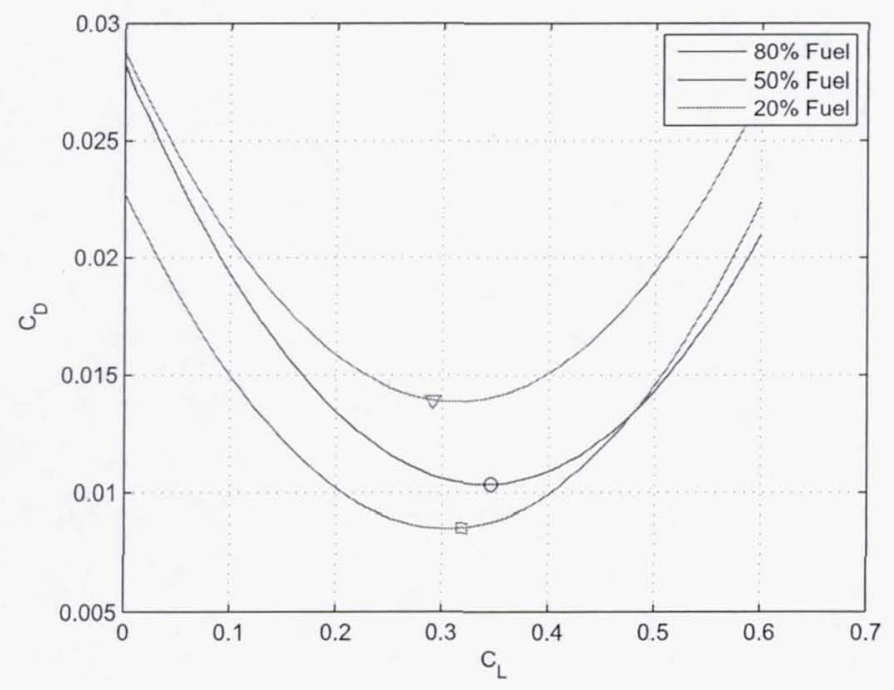

Figure 36 - Induced $C_{D_{l}}$ Penalty due to Aeroelastic Deflection for Drooped-Wing Aircraft

Let

- $C_{L}$ and $C_{D_{i}}$ be the values corresponding to the drooped-wing aircraft configuration, where $C_{D_{i}}$ is the induced drag component 
- $C_{L_{f}}$ and $C_{D_{f}}$ be the values corresponding to the drooped-wing aircraft configuration with wing shaping control using variable camber continuous trailing edge flap system which results in incremental $\Delta C_{L_{f}}$ and $\Delta C_{D_{f}}$ values which are assumed to be linearly varying with flap deflections

- $C_{L}^{*}$ and $C_{D_{i}}^{*}$ be the values corresponding to the baseline aircraft

- $C_{D_{0}}$ be the parasitic drag due to skin friction

Table 7 shows the lift and drag coefficients as a function of the fuel burn and with aeroelastic deflections taken into account

\begin{tabular}{|c||c|c|c|c|c|c|c|c|c|c|}
\hline Fuel Ratio & $C_{L}$ & $C_{D_{0}}$ & $C_{D_{i}}$ & $C_{D}$ & $C_{D_{i}}^{*}$ & $C_{D}^{*}$ & $\Delta C_{L_{f}}$ & $C_{L_{f}}$ & $\Delta C_{D_{f}}$ & $C_{D_{f}}$ \\
\hline \hline 0.2 & 0.2913 & 0.00941 & 0.01393 & 0.02334 & 0.01530 & 0.02470 & 0.1347 & 0.4260 & 0.00234 & 0.02026 \\
\hline 0.3 & 0.3004 & 0.00941 & 0.01371 & 0.02312 & 0.01533 & 0.02474 & 0.0898 & 0.3902 & 0.00156 & 0.01946 \\
\hline 0.4 & 0.3095 & 0.00941 & 0.01358 & 0.02299 & 0.01539 & 0.02480 & 0.0449 & 0.3544 & 0.00078 & 0.01868 \\
\hline 0.5 & 0.3186 & 0.00941 & 0.00852 & 0.01793 & 0.01541 & 0.02482 & 0 & 0.3186 & 0 & 0.01793 \\
\hline 0.6 & 0.3277 & 0.00941 & 0.01051 & 0.01992 & 0.01543 & 0.02484 & -0.0426 & 0.2851 & 0.00072 & 0.01870 \\
\hline 0.7 & 0.3368 & 0.00941 & 0.01042 & 0.01983 & 0.01552 & 0.02493 & -0.0852 & 0.2516 & 0.00144 & 0.01950 \\
\hline 0.8 & 0.3459 & 0.00941 & 0.01033 & 0.01974 & 0.01557 & 0.02498 & -0.1278 & 0.2180 & 0.00216 & 0.02033 \\
\hline
\end{tabular}

Table 7 - $C_{L}$ and $C_{D}$ due to Aeroelastic Deflection and Fuel Burn

Clearly wing shaping control would be beneficial if the drag penalty due to the flap and slat deflections does not exceed that due to a non-optimal wing shape. It is also clear that the conventional flap and slat systems are not beneficial for wing shaping control because of its large drag penalty. Table 7 shows that the variable camber continuous trailing edge flaps are not beneficial until the fuel point reaches about $70 \%$. However, for the flap down configuration when the fuel is less than half full, the variable camber continuous trailing edge flaps become quite beneficial as the $C_{D_{f}}$ values are significantly less than the $C_{D_{i}}$ values. Comparing the $C_{D_{f}}$ values with the baseline $C_{D_{i}}^{*}$ values, the drag reduction of the wing shaping control of the drooped-wing aircraft configuration is quite apparent. The large $C_{D_{i}}^{*}$ values are due to the adverse effect of aeroelastic deflections. Also since the drooped-wing aircraft configuration includes a 1-g aeroelastic deflection in the as-built configuration, the adverse effect of aeroelastic deflections is minimized for the drooped-wing aircraft configuration.

\subsection{Cruise Analysis}

During cruise, fuel is burned by the engines that causes a change in the aircraft weight according to the weight equation

$$
\dot{W}=-c T
$$

where $T$ is the engine thrust and $c$ is the Thrust Specific Fuel Consumption (TSFC) which is equal to $0.5295 / \mathrm{hr}$ for Mach 0.8 and $30,000 \mathrm{ft}$ according to Eq. (2.2).

Range and endurance are two performance parameters for an aircraft in a cruise phase. Range is defined as the distance that an aircraft can fly for a given amount of fuel available. The range can be computed from the Breguet range equation [14]

$$
r=-\int_{W_{t}}^{W_{f}} \frac{V}{c T} d W
$$

In cruise, drag is equal to thrust and lift equal to weight. Then the range equation can be written as

$$
r=-\int_{W_{t}}^{W_{f}} \frac{V}{c}\left(\frac{C_{L}}{C_{D}}\right) \frac{d W}{W}
$$

Thus the range is proportional to the aerodynamic efficiency which is defined as the lift-to-drag ratio or $E=C_{L} / C_{D}$. Table 8 shows the value of the aerodynamic efficiency. 


\begin{tabular}{|c||c|c|c|}
\hline Fuel Ratio & $E$ & $E^{*}$ & $E_{f}$ \\
\hline \hline 0.2 & 12.4807 & 11.7935 & 21.0267 \\
\hline 0.3 & 12.9931 & 12.1423 & 20.0514 \\
\hline 0.4 & 13.4624 & 12.4798 & 18.9722 \\
\hline 0.5 & 17.7691 & 12.8364 & 17.7691 \\
\hline 0.6 & 16.4508 & 13.1924 & 15.2460 \\
\hline 0.7 & 16.9844 & 13.5098 & 12.9026 \\
\hline 0.8 & 17.5228 & 13.8471 & 10.7231 \\
\hline
\end{tabular}

Table 8- Aerodynamic Efficiency

An interesting observation is made in that the aerodynamic efficiency for the flap-up configuration when the fuel is more than $50 \%$ is lower than the no-flap configuration. Thus, there is no advantage for wing shaping control when the flaps are configured in the upward position. On the other hand, the downward deflection of flaps is highly beneficial in increasing the aerodynamic efficiency. Hence, for the cruise analysis, it will be assumed that wing shaping control will only be used when the fuel is less than half full in the tank.

Figure 37 is a plot of three different cruise ranges for the three aircraft configurations. The drooped-wing aircraft configuration with no wing shaping control provides a significant benefit of fuel savings. At a first glance, it appears that the fuel savings with the drooped-wing aircraft configuration are huge as compared to the baseline aircraft . However, the comparison may not be on the same basis since the drooped-wing aircraft configuration has an advantage of the aeroelastic tailoring by incorporating the $1-\mathrm{g}$ aeroelastic deflection into the as-built configuration. If this aeroelastic tailoring is also applied to the baseline aircraft, then the fuel savings will be less and probably will be in the ball park of the $5.3 \%$ drag reduction.

Comparing between the drooped-wing aircraft configuration with and without wing shaping control, The advantage of wing shaping control can clearly be demonstrated. For a $4000-1 b$ fuel burn, a fuel saving of $17 \%$ is realized due to wing shaping control. This result indicates the potential benefits of wing shaping control for long range cruises. For short range cruises with the fuel burn less than half of the fuel, there may be no benefit of wing shaping control. Thus, in summary, the drag reduction benefits of both the drooped-wing shape and the variable camber continuous trailing edge flap can result in substantial fuel savings. The result thus merits a further investigation as part of a follow-on effort to develop the concepts in a more-in-depth study.

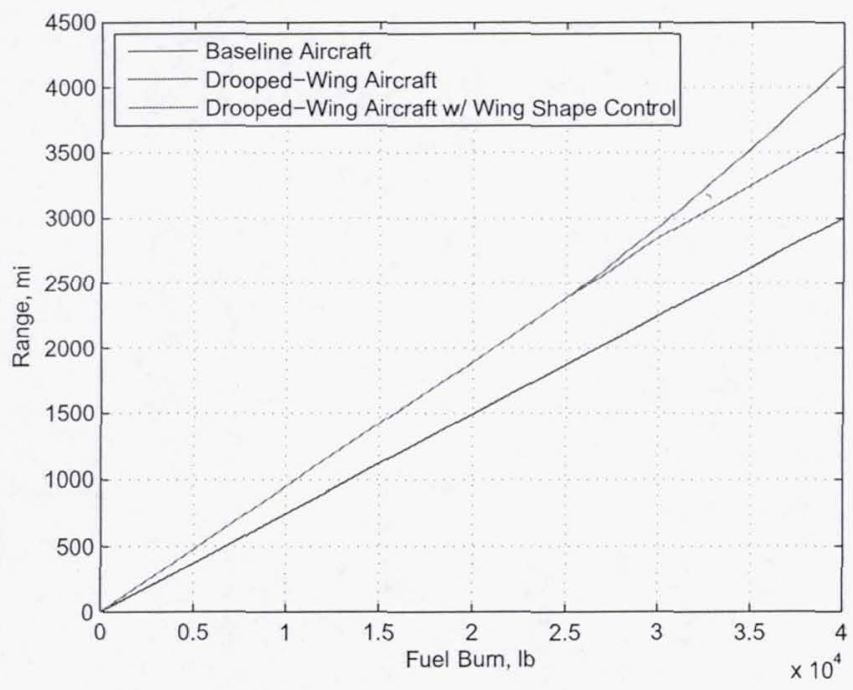

Figure 37 - Comparison of Cruise Ranges 


\section{Aeroelastic Analysis}

The elastic shaped aircraft concept is assumed to have flexible wings that can be elastically shaped in-flight by wing shaping control. In order to develop active wing shaping control, the effect of structural flexibility of the wing on aircraft flight dynamics must be considered. Aeroelasticity theory is used to develop an aeroelastic flight dynamic model of the elastically shaped aircraft concept to account for interactions between wing bending and torsion on aircraft performance and stability.

\subsection{Reference Frames}

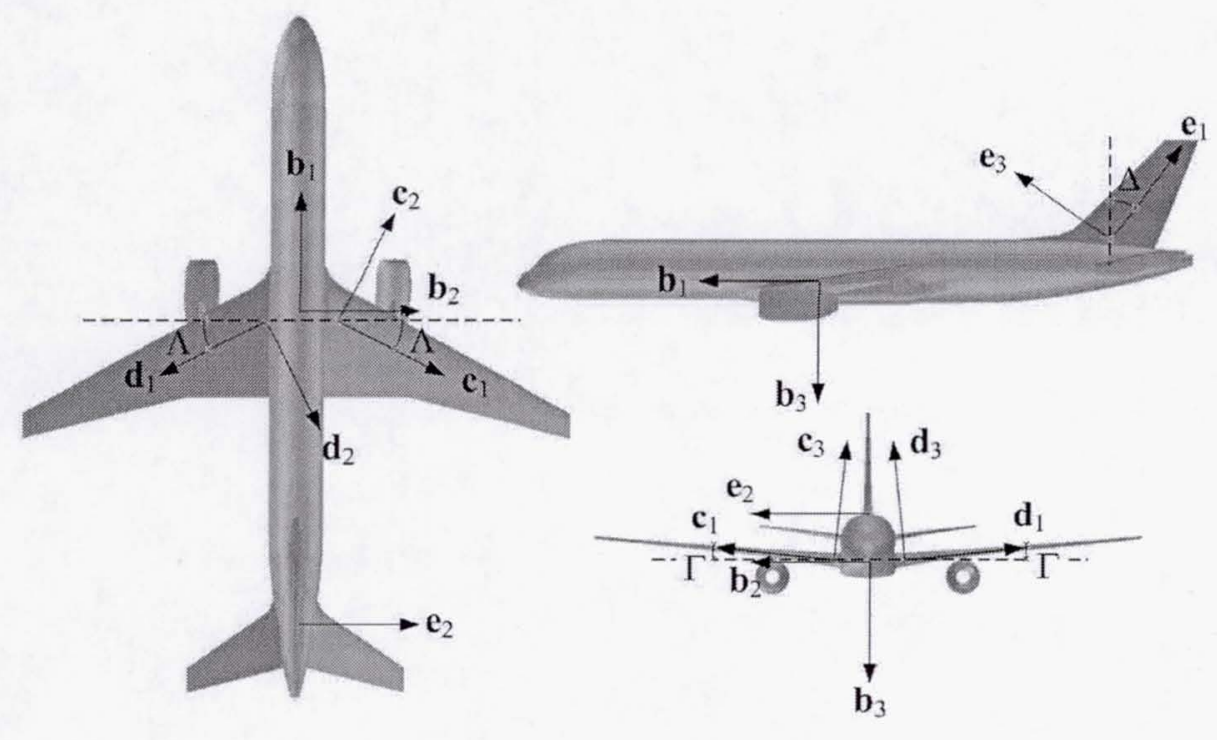

Figure 38 - Aircraft Reference Frames

Figure 38 illustrates three orthogonal views of a typical aircraft. Several reference frames are introduced to facilitate the rigid-body dynamic and structural dynamic analysis of the lifting surfaces. For example, the aircraft inertial reference frame $A$ is defined by unit vectors $\mathbf{a}_{1}, \mathbf{a}_{2}$, and $\mathbf{a}_{3}$ fixed to the non-rotating earth. The aircraft body-fixed reference frame $B$ is defined by unit vectors $\mathbf{b}_{1}, \mathbf{b}_{2}$, and $\mathbf{b}_{3}$. The reference frames $A$ and $B$ are related by three successive rotations: 1) the first rotation about $\mathbf{a}_{3}$ by the heading angle $\psi$ that results in an intermediate reference frame $\mathrm{A}^{\prime}$ defined by unit vectors $\mathbf{a}_{1}^{\prime}, \mathbf{a}_{2}^{\prime}$, and $\mathbf{a}_{3}^{\prime}$ (not shown), 2) the second rotation about $\mathbf{a}_{2}^{\prime}$ by the pitch angle $\theta$ that results in an intermediate reference frame $\mathrm{B}^{\prime}$ defined by unit vectors $\mathbf{b}_{1}^{\prime}, \mathbf{b}_{2}^{\prime}$, and $\mathbf{b}_{3}^{\prime}$ (not shown), and 3) the third rotation about $\mathbf{b}_{1}^{\prime}$ by the bank angle $\phi$ that results in the reference frame $\mathrm{B}$. This relationship can be expressed as

$$
\begin{aligned}
{\left[\begin{array}{l}
\mathbf{a}_{1} \\
\mathbf{a}_{2} \\
\mathbf{a}_{3}
\end{array}\right]=} & {\left[\begin{array}{ccc}
\cos \psi & -\sin \psi & 0 \\
\sin \psi & \cos \psi & 0 \\
0 & 0 & 1
\end{array}\right]\left[\begin{array}{ccc}
\cos \theta & 0 & \sin \theta \\
0 & 1 & 0 \\
-\sin \theta & 0 & \cos \theta
\end{array}\right]\left[\begin{array}{ccc}
1 & 0 & 0 \\
0 & \cos \phi & -\sin \phi \\
0 & \sin \phi & \cos \phi
\end{array}\right]\left[\begin{array}{l}
\mathbf{b}_{1} \\
\mathbf{b}_{2} \\
\mathbf{b}_{3}
\end{array}\right] } \\
= & {\left[\begin{array}{ccc}
\cos \psi \cos \theta & -\sin \psi \cos \phi+\cos \psi \sin \theta \sin \phi & \sin \psi \sin \phi+\cos \psi \sin \theta \cos \phi \\
\sin \psi \cos \theta & \cos \psi \cos \phi+\sin \psi \sin \theta \sin \phi & -\cos \psi \sin \phi+\sin \psi \sin \theta \cos \phi \\
-\sin \theta & \cos \theta \sin \phi & \cos \theta \cos \phi
\end{array}\right]\left[\begin{array}{l}
\mathbf{b}_{1} \\
\mathbf{b}_{2} \\
\mathbf{b}_{3}
\end{array}\right] }
\end{aligned}
$$

The left wing elastic reference frame $D$ is defined by unit vectors $\mathbf{d}_{1}, \mathbf{d}_{2}$, and $\mathbf{d}_{3}$. The reference frames $B$ and $\mathrm{D}$ are related by three successive rotations: 1) the first rotation about $\mathbf{b}_{3}$ by the elastic axis sweep angle $\frac{3 \pi}{2}-\Lambda$ that results in an intermediate reference frame B" defined by unit vectors $\mathbf{b}_{1}^{\prime \prime}, \mathbf{b}_{2}^{\prime \prime}$, and $\mathbf{b}_{3}^{\prime \prime}$ (not shown), 2) the second rotation about negative $\mathbf{b}_{2}^{\prime \prime}$ by the elastic axis dihedral angle $\Gamma$ that results in an intermediate reference frame $D^{\prime}$ defined by unit vectors $\mathbf{d}_{1}^{\prime}, \mathbf{d}_{2}^{\prime}$, and $\mathbf{d}_{3}^{\prime}$ (not shown), and 3) the third rotation about $\mathbf{d}_{1}^{\prime}$ by an angle $\pi$ that results in the reference frame 
D. This relationship can be expressed as

$$
\begin{aligned}
{\left[\begin{array}{l}
\mathbf{b}_{1} \\
\mathbf{b}_{2} \\
\mathbf{b}_{3}
\end{array}\right]=\left[\begin{array}{ccc}
-\sin \Lambda & \cos \Lambda & 0 \\
-\cos \Lambda & -\sin \Lambda & 0 \\
0 & 0 & 1
\end{array}\right]\left[\begin{array}{ccc}
\cos \Gamma & 0 & -\sin \Gamma \\
0 & 1 & 0 \\
\sin \Gamma & 0 & \cos \Gamma
\end{array}\right]\left[\begin{array}{ccc}
1 & 0 & 0 \\
0 & -1 & 0 \\
0 & 0 & -1
\end{array}\right]\left[\begin{array}{l}
\mathbf{d}_{1} \\
\mathbf{d}_{2} \\
\mathbf{d}_{3}
\end{array}\right] } \\
= \\
=\left[\begin{array}{ccc}
-\sin \Lambda \cos \Gamma & -\cos \Lambda & \sin \Lambda \sin \Gamma \\
-\cos \Lambda \cos \Gamma & \sin \Lambda & \cos \Lambda \sin \Gamma \\
-\sin \Gamma & 0 & -\cos \Gamma
\end{array}\right]\left[\begin{array}{l}
\mathbf{d}_{1} \\
\mathbf{d}_{2} \\
\mathbf{d}_{3}
\end{array}\right]
\end{aligned}
$$

Generally, the effect of the dihedral angle can be significant. A full analysis with the dihedral angle can be performed but can also result in a very complex analytical formulation. Thus, to simplify the analysis, the dihedral effect is assumed to be negligible in this study. The right wing reference frame $\mathrm{C}$ can be established in a similar manner. In the analysis, the aeroelastic effects on the fuselage, horizontal stabilizers, and vertical stabilizer are not considered, but the analytical method can be formulated for analyzing these lifting surfaces if necessary. In general, a whole aircraft analysis approach should be conducted to provide a comprehensive assessment of the effect of structural flexibility on aircraft performance and stability. However, the scope of this study pertains to only the wing structures.

\subsection{Elastic Analysis}

In the subsequent analysis, the combined motion of the left wing is considered. The wing has a varying pre-twist angle $\gamma(x)$ common in many aircraft. Typically, the wing pre-twist angle varies from being nose-up at the wing root to nose-down at the wing tip. The nose-down pre-twist at the wing tip is designed to delay stall onsets. This is called a wash-out twist distribution. Under aerodynamic forces and moments, wing structural deflections introduce strains in the wing structure. For high aspect ratio wings, an equivalent beam approach can be used to analyze structural deflections with a reasonable accuracy. The equivalent beam approach is a typical formulation in many aeroelasticity studies [15]. Experimental validation can show that equivalent beam approach is accurate for an aspect ratio as low as 3:1. The internal structure of a wing typically comprises a complex arrangement of load carrying spars and wing boxes. Nonetheless, the elastic behavior of a wing can be captured by the use of equivalent stiffness properties. These properties can be derived from structural certification testing that yields information about wing deflection as a function of loading. It is assumed that the effect of wing curvature is ignored and the straight beam theory is used to model the wing deflection.

Consider an airfoil section on the left wing as shown in Figure 39 undergoing bending and twist deflections.

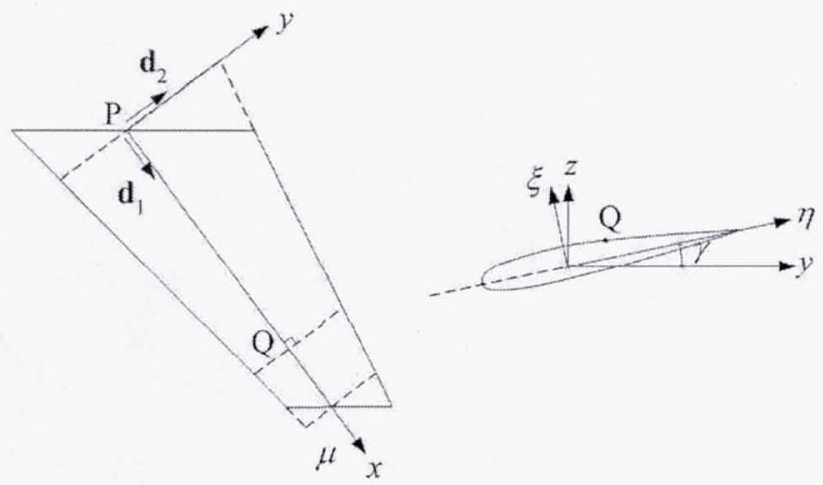

Figure 39 - Left Wing Reference Frame

Let $(x, y, z)$ be the coordinates of a point $\mathrm{Q}$ on the airfoil. Then

$$
\left[\begin{array}{l}
y \\
z
\end{array}\right]=\left[\begin{array}{cc}
\cos \gamma & -\sin \gamma \\
\sin \gamma & \cos \gamma
\end{array}\right]\left[\begin{array}{l}
\eta \\
\xi
\end{array}\right]
$$

where $\eta$ and $\xi$ are local airfoil coordinates, and $\gamma$ is the wing section pre-twist angle, positive nose-down [16]. 
The axial or extensional deffection of a wing is generally very small and therefore can usually be neglected. Let $V$ and $W$ be chordwise and flapwise bending deflections of point Q, respectively, and let $\Theta$ be a torsional twist angle about the $x$-axis, positive nose-down. Then, the rotation angle due to the structural deformation can be expressed as

$$
\phi(x, t)=\Theta \mathbf{d}_{1}-W_{x} \mathbf{d}_{2}+V_{x} \mathbf{d}_{3}
$$

where the subscripts $x$ and $t$ denote the partial derivatives of $V, W$, and $\Theta$.

Let $\left(x_{1}, y_{1}, z_{1}\right)$ be the coordinates of point $\mathrm{Q}$ on the airfoil in the reference frame $\mathrm{D}$. Then the coordinates $\left(x_{1}, y_{1}, z_{1}\right)$ are computed using the small angle approximation as [17]

$$
\left[\begin{array}{c}
x_{1}(x, t) \\
y_{1}(x, t) \\
z_{1}(x, t)
\end{array}\right]=\left[\begin{array}{c}
x \\
y+V \\
z+W
\end{array}\right]+\left[\begin{array}{c}
\phi \times\left(y \mathbf{d}_{2}+z \mathbf{d}_{3}\right) \cdot \mathbf{d}_{1} \\
\phi \times\left(y \mathbf{d}_{2}+z \mathbf{d}_{3}\right) \cdot \mathbf{d}_{2} \\
\phi \times\left(y \mathbf{d}_{2}+z \mathbf{d}_{3}\right) \cdot \mathbf{d}_{3}
\end{array}\right]=\left[\begin{array}{c}
x-y V_{x}-z W_{x} \\
y+V-z \Theta \\
z+W+y \Theta
\end{array}\right]
$$

Differentiating $x_{1}, y_{1}$, and $z_{1}$ with respect to $x$ yields

$$
\left[\begin{array}{c}
x_{1, x} \\
y_{1, x} \\
z_{1, x}
\end{array}\right]=\left[\begin{array}{c}
1-y V_{x x}+z \gamma^{\prime} V_{x}-z W_{x x}-y \gamma^{\prime} W_{x} \\
-z \gamma^{\prime}+V_{x}-z \Theta_{x}-y \gamma^{\prime} \Theta \\
y \gamma^{\prime}+W_{x}+y \Theta_{x}-z \gamma^{\prime} \Theta
\end{array}\right]
$$

Neglecting the transverse shear effect, the longitudinal strain is computed as

$$
\varepsilon=\frac{d s_{1}-d s}{d s}=\frac{s_{1, x}}{s_{x}}-1
$$

where

$$
\begin{gathered}
s_{x}=\sqrt{1+y_{x}^{2}+z_{x}^{2}}=\sqrt{1+\left(y^{2}+z^{2}\right)\left(\gamma^{\prime}\right)^{2}} \\
s_{1, x}=\sqrt{x_{1, x}^{2}+y_{1, x}^{2}+z_{1, x}^{2}}=\sqrt{1+\left(y^{2}+z^{2}\right)\left(\gamma^{\prime}\right)^{2}-2 y V_{x x}-2 z W_{x x}+2\left(y^{2}+z^{2}\right) \gamma^{\prime} \Theta_{x}}
\end{gathered}
$$

For a small wing twist angle $\gamma$, the longitudinal strain is obtained as [17]

$$
\varepsilon=-y V_{x x}-z W_{x x}+\left(y^{2}+z^{2}\right) \gamma^{\prime} \Theta_{x}
$$

The moments acting on the wing are then obtained as

$$
\left[\begin{array}{c}
M_{x} \\
M_{y} \\
M_{z}
\end{array}\right]=\left[\begin{array}{c}
G J \Theta_{x} \\
0 \\
0
\end{array}\right]+\iint E \varepsilon\left[\begin{array}{c}
\left(y^{2}+z^{2}\right)\left(\gamma^{\prime}+\Theta_{x}\right) \\
-z \\
-y
\end{array}\right] d y d z=\left[\begin{array}{ccc}
G J+E B_{1}\left(\gamma^{\prime}\right)^{2} & -E B_{2} \gamma^{\prime} & -E B_{3} \gamma^{\prime} \\
-E B_{2} \gamma^{\prime} & E I_{y y} & -E I_{y z} \\
-E B_{3} \gamma^{\prime} & -E I_{y z} & E I_{z z}
\end{array}\right]\left[\begin{array}{c}
\Theta_{x} \\
W_{x x} \\
V_{x x}
\end{array}\right]
$$

where $E$ is the Young's modulus; $G$ is the shear modulus; $\gamma$ ' is the derivative of the wing pre-twist angle; $I_{y y}, I_{y z}$, and $I_{z z}$ are the section area moments of inertia about the flapwise axis; $J$ is the torsional constant; and $B_{1}, B_{2}$, and $B_{3}$ are the bending-torsion coupling constants which are defined as

$$
\left[\begin{array}{l}
B_{1} \\
B_{2} \\
B_{3}
\end{array}\right]=\iint\left(y^{2}+z^{2}\right)\left[\begin{array}{c}
y^{2}+z^{2} \\
z \\
y
\end{array}\right] d y d z
$$

The strain analysis shows that for a pre-twisted wing the bending deflections $V$ and $W$ are coupled to the torsional deflection $\Theta$ via the slope of the wing pre-twist angle. This coupling can be significant if the term $\gamma^{\prime}$ is dominant as in highly twisted wings such as turbomachinery blades. For an aircraft wing structure, a simplification can be made by neglecting the chordwise bending deflection. Thus, the resulting moments are now given as

$$
\left[\begin{array}{c}
M_{x} \\
M_{y}
\end{array}\right]=\left[\begin{array}{cc}
G J+E B_{1}\left(\gamma^{\prime}\right)^{2} & -E B_{2} \gamma^{\prime} \\
-E B_{2} \gamma^{\prime} & E I_{y y}
\end{array}\right]\left[\begin{array}{c}
\Theta_{x} \\
W_{x x}
\end{array}\right]
$$




\subsection{Structural Stiffness Estimates}

Structural information for aircraft is generally not publicly available. Therefore, the uncertainty on the structural analysis can be high. Nonetheless, certain assumptions can be made for the structural stiffness of a wing if the wing deflection is known. In the study, the wing deflection for the baseline vehicle at 1-g loading at cruise is assumed to be about $3 \mathrm{ft}$ at the tip. This is based on an observation of the result of a structural certification test on a Boeing 777 wing. This wing deflected at the tip about $24 \mathrm{ft}$ with an applied load of $3.75 \mathrm{~g}$ 's. This corresponds to 1.5 times the design load which is established at $2.5 \mathrm{~g}$ 's. The wing span of Boeing 777 is $200 \mathrm{ft}$. So the deflection at 1-g loading is estimated to be about $6.4 \mathrm{ft}$ by scaling the deflection by the loading ratio. The wing tip deflection for a cantilever beam with a different length $L$ and structural stiffness $E I$ is proportional to $L^{3} / E I$. Assuming that $E I$ is proportional to $L^{3 / 2}$. Then, the wing tip deflection is proportional to $L^{3 / 2}$. Using this approximation, the wing tip deflection at $1-\mathrm{g}$ loading for the baseline aircraft wing is estimated to be about $3 \mathrm{ft}$.
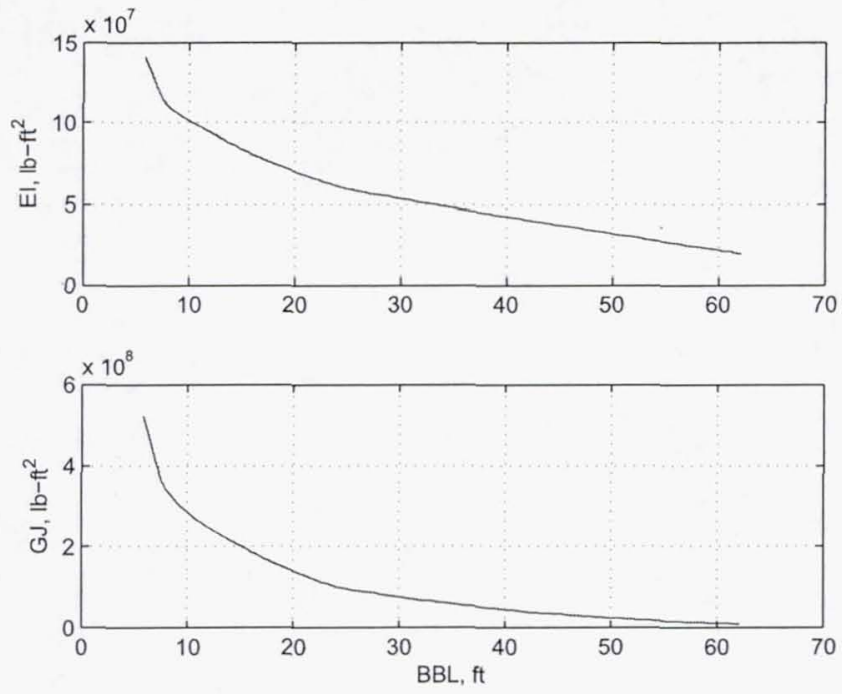

Figure 40 - Estimated Wing Bending and Torsional Stiffness
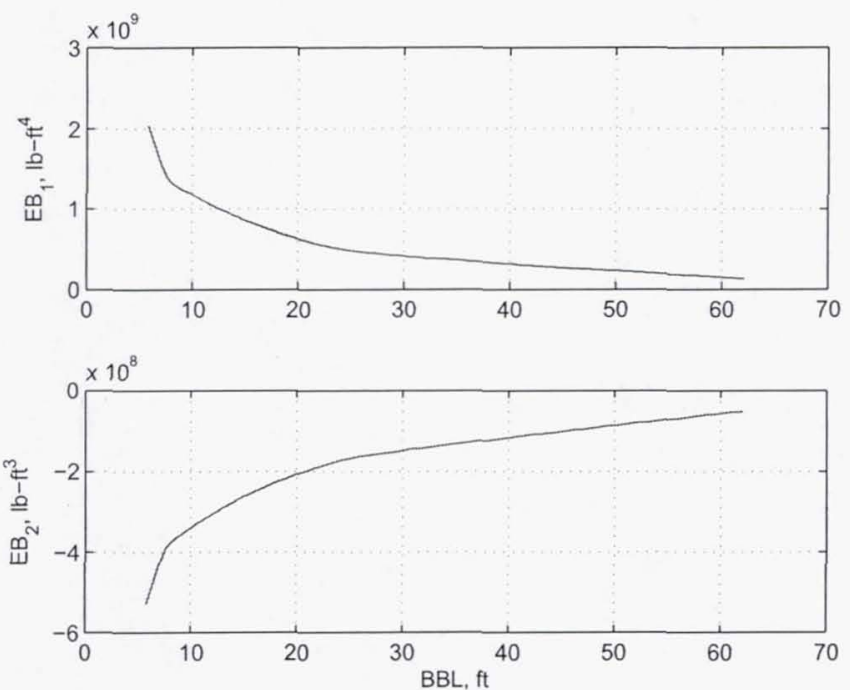

Figure 41 - Estimated Wing Bending-Torsion Coupling Stiffness 
The wing is modeled as a thin-walled shell structure for which the section properties are computed. The Young's modulus and shear modulus are then adjusted by trial and error until the desired wing tip deflection is obtained. The distributions of the wing structural stiffness characteristics are plotted in Figures 40 and 41.

\subsection{Aeroelastic Angle of Attack}

The relative velocity of the air approaching a wing section includes the contribution from the wing structural deflection that results in changes in the local angle of attack. Since aerodynamic forces and moments are dependent on the local angle of attack, the wing structural deflection will generate additional elastic forces and moments. The local angle of attack depends on the relative approaching air velocity as well as the rotation angle $\phi$ from Eq. (5.4). The relative air velocity in turn also depends on a structural-deflection induced velocity. The local velocity components at point $\mathrm{Q}$ in the reference frame $\mathrm{D}$ are given by

$$
\left[\begin{array}{c}
v_{x} \\
v_{y} \\
v_{z}
\end{array}\right]=\left[\begin{array}{c}
-u \sin \Lambda+x_{1, t} \\
-u \cos \Lambda+y_{1, t} \\
-w-q x_{a}+z_{1, t}
\end{array}\right]=\left[\begin{array}{c}
-u \sin \Lambda-z W_{x t} \\
-u \cos \Lambda-z \Theta_{t} \\
-w-q x_{a}+W_{t}+y \Theta_{t}
\end{array}\right]
$$

where $u \approx V_{\infty}, w \approx V_{\infty} \alpha, q$ is the aircraft pitch rate, $x_{a}$ is the position of point Q with respect to the aircraft C.G. (positive aft of C.G.) measured in the aircraft stability reference frame B, and $y$ and $z$ are coordinates of point $Q$ in the reference frame D.

In order to compute the aeroelastic forces and moments, the velocity must be transformed from the reference frame $\mathrm{D}$ to the airfoil local coordinate reference frame defined by $(\mu, \eta, \xi)$ (see Figure 5.2). Then the transformation can be performed using two successive rotation matrix multiplication operations as

$$
\left[\begin{array}{l}
v_{\mu} \\
v_{\eta} \\
v_{\xi}
\end{array}\right]=\left[\begin{array}{ccc}
1 & 0 & 0 \\
0 & 1 & \Theta+\gamma \\
0 & -(\Theta+\gamma) & 1
\end{array}\right]\left[\begin{array}{ccc}
1 & 0 & W_{x} \\
0 & 1 & 0 \\
-W_{x} & 0 & 1
\end{array}\right]\left[\begin{array}{c}
v_{x} \\
v_{y} \\
v_{z}
\end{array}\right]=\left[\begin{array}{c}
v_{x}+W_{x} v_{z} \\
v_{y}+(\Theta+\gamma)\left(v_{z}-W_{x} v_{x}\right) \\
v_{z}-W_{x} v_{x}-(\Theta+\gamma) v_{y}
\end{array}\right]
$$

Referring to Figure 42, the local aeroelastic angle of attack on the airfoil section is due to the velocity components $v_{\eta}$ and $v_{\xi}$ and is computed as

$$
\alpha_{c}=\frac{v_{\xi}}{v_{\eta}}
$$

where

$$
\begin{gathered}
v_{\xi} \approx w+q x_{a}-W_{t}-y \Theta_{t}-W_{x} u \sin \Lambda-(\Theta+\gamma) u \cos \Lambda \\
v_{\eta} \approx u \cos \Lambda
\end{gathered}
$$

Assuming $z \approx 0$, then the local aeroelastic angle of attack can be expressed as a function of $x$ and $y$ [16]

$$
\alpha_{c}(x, y)=\frac{\alpha}{\cos \Lambda}+\frac{q x_{a}}{V_{\infty} \cos \Lambda}-\gamma-W_{x} \tan \Lambda-\Theta-\frac{W_{t}+y \Theta_{t}}{V_{\infty} \cos \Lambda}
$$

The terms $W_{t}$ and $\Theta_{t}$ contribute to aerodynamic damping forces which can be significant for aeroelastic stability.

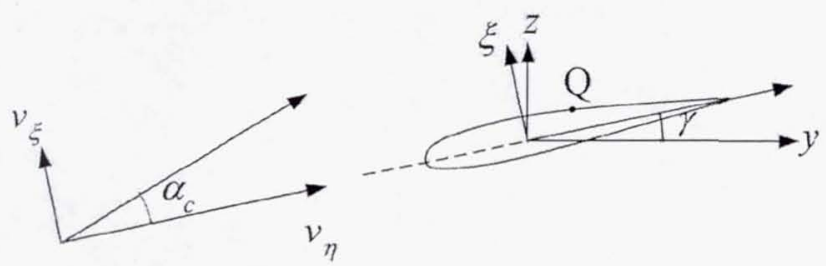

Figure 42 - Aeroelastic Angle of Attack 
There are two methods for aeroelastic analysis. The quasi-steady aerodynamic method assumes that the lift circulation is established instantaneously. The Theodorsen's aeroelasticity theory accounts for aerodynamic lag effects due to unsteady lift circulation[16]. For steady state aerodynamics, the Theodorsen's theory tends to the quasi-steady aerodynamics. The local angle of attack of an airfoil section at the aerodynamic center due to lift circulation is evaluated with $y=-e$ as

$$
\alpha_{a c}=\frac{\alpha}{\cos \Lambda}+\frac{q x_{a c}}{V_{\infty} \cos \Lambda}-\gamma-W_{x} \tan \Lambda-\Theta-\frac{W_{t}-e \Theta_{t}}{V_{\infty} \cos \Lambda}
$$

where $x_{a c}$ is the forward distance of the aircraft center of gravity from the wing section aerodynamic center measured in aircraft stability reference frame (positive aft of C.G.) and $e$ is the forward distance of the aerodynamic center from the elastic center.

There is another source of lift, called non-circulatory lift due to the reaction force of the air volume surrounding a wing section. The non-circulatory lift force is based on the aeroelastic angle of attack at the mid-chord location where $y=e_{m}$

$$
\alpha_{m c}=\frac{\alpha}{\cos \Lambda}+\frac{q x_{m}}{V_{\infty} \cos \Lambda}-\gamma-W_{x} \tan \Lambda-\Theta-\frac{W_{t}+e_{m} \Theta_{t}}{V_{\infty} \cos \Lambda}
$$

where $x_{m}$ is the forward distance of the aircraft center of gravity from the mid-chord location of a wing section and $e_{m}$ is the forward distance of the aeroelastic center from the mid-chord location.

\subsection{Wing Aeroelasticity}

The wing shape is comprised of two components: bending deflection $W(x)$ and torsional deflection $\Theta(x)$, where $x$ is the wing local elastic axis. Furthermore, these deflections include both the static and dynamic contributions.

The force and moment equilibrium conditions for bending and torsion are expressed as

$$
\begin{gathered}
\frac{\partial M_{x}}{\partial x}=-m_{x} \\
\frac{\partial^{2} M_{y}}{\partial x^{2}}=f_{z}-\frac{\partial m_{y}}{\partial x}
\end{gathered}
$$

where $m_{x}$ is the pitching moment per unit span about the elastic axis, $f_{z}$ is the lift force per unit span, and $m_{y}$ is the bending moment per unit span about the flapwise axis of the wing which is assumed to be zero.

The wing section lift coefficient is given by

$$
c_{L_{a c}}=c_{L_{\alpha}} C(k) \alpha_{a c}+c_{L_{\delta}} \delta
$$

where $k=\frac{\omega c}{2 V_{\infty}}$ is the reduced frequency parameter, $\omega$ is the frequency of wing oscillations, $c$ is the section chord, $c_{L_{\alpha}}$ is the section lift curve slope, $c_{L_{\delta}}$ is the section lift derivative, and $\delta$ is the control surface deflection.

The function $C(k)$ is the Theodorsen's complex-valued function which is also expressed in terms of Bessel functions as

$$
C(k)=F(k)+i G(k)
$$

where $F(k)>0$ and $G(k)<0$.

The wing section lift coefficient due to harmonic motions is expressed as

$$
c_{L_{a c}}=c_{L_{\alpha}} \alpha_{a c} F(k)+c_{L_{\alpha}} \frac{\dot{\alpha}_{a c} c}{2 V_{\infty}} \frac{G(k)}{k}+c_{L_{\delta}} \delta
$$

In addition, the apparent mass of the air contributes to the lift force acting at the mid-chord location as follows:

$$
c_{L_{m c}}=\frac{\pi \dot{\alpha}_{m c} c}{2 V_{\infty}}
$$

The total section lift coefficient is

$$
c_{L}=c_{L_{a c}}+c_{L_{m c}}
$$


The section pitching moment coefficient is evaluated as

$$
c_{m}=c_{m_{a c}}+\frac{e}{c} c_{L_{a c}}-\frac{e_{m}}{c} c_{L_{m c}}+c_{m_{\delta}} \delta
$$

where $c_{m_{a c}}$ is the section pitching moment coefficient at the aerodynamic center and $c_{m_{\delta}}$ is the section pitching moment derivative at the aerodynamic center due to the control surface deflection $\delta$.

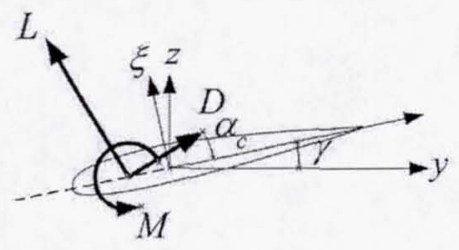

Figure 43 - Airfoil Forces and Moment

Using the sign convention as shown in Figure 43, the pitching moment per unit span can now be expressed as

$$
m_{x}=-c_{m} q_{\infty} \cos ^{2} \Lambda c^{2}+m g e_{c g}-m k^{2} \Theta_{t t}+m e_{c g} W_{t t}
$$

where $q_{\infty}$ is the dynamic pressure, $m$ is the wing mass distribution including fuel mass, $k$ is the wing section radius of gyration, and $e_{c g}$ is the forward distance of the elastic center from the center of mass,

The lift force per unit span is given by

$$
f_{z}=c_{L} q_{\infty} \cos ^{2} \Lambda c-m g-m W_{t t}+m e_{c g} \Theta_{t t}
$$

where $A$ is the cross sectional area of a wing section.

The aeroservoelastic bending and torsional equations then become

$$
\begin{aligned}
& \frac{\partial}{\partial x}\left\{\left[G J+E B_{1}\left(\gamma^{\prime}\right)^{2}\right] \Theta_{x}-E B_{2} \gamma^{\prime} W_{x x}\right\}=\left[c c_{m_{a c}}+e c_{L_{\alpha}}\left(\frac{\alpha}{\cos \Lambda}-\gamma+\frac{x_{a} q}{V_{\infty} \cos \Lambda}-W_{x} \tan \Lambda-\Theta-\frac{W_{t}-e \Theta_{t}}{V_{\infty} \cos \Lambda}\right) F(k)\right. \\
& +e c_{L_{\alpha}}\left(\frac{\dot{\alpha}}{\cos \Lambda}-W_{x t} \tan \Lambda-\Theta_{t}-\frac{W_{t t}-e \Theta_{t t}}{V_{\infty} \cos \Lambda}\right) \frac{c}{2 V_{\infty}} \frac{G(k)}{k}+\left(c c_{m_{\delta}}+e c_{L_{\delta}}\right) \delta \\
& \left.-\frac{\pi c e_{m}}{2 V_{\infty}}\left(\frac{\dot{\alpha}}{\cos \Lambda}-W_{x t} \tan \Lambda-\Theta_{t}-\frac{W_{t t}+e_{m} \Theta_{t t}}{V_{\infty} \cos \Lambda}\right)\right] q_{\infty} \cos ^{2} \Lambda c-m g e_{c g}+m k^{2} \Theta_{t t}-m e_{c g} W_{t t} \\
& \frac{\partial^{2}}{\partial x^{2}}\left(-E B_{2} \gamma^{\prime} \Theta_{x}+E I_{y y} W_{x x}\right)=\left[c_{L_{\alpha}}\left(\frac{\alpha}{\cos \Lambda}-\gamma+\frac{x_{a} q}{V_{\infty} \cos \Lambda}-W_{x} \tan \Lambda-\Theta-\frac{W_{t}-e \Theta_{t}}{V_{\infty} \cos \Lambda}\right) F(k)\right. \\
& +c_{L_{\alpha}}\left(\frac{\dot{\alpha}}{\cos \Lambda}-W_{x t} \tan \Lambda-\Theta_{t}-\frac{W_{t t}-e \Theta_{t t}}{V_{\infty} \cos \Lambda}\right) \frac{c}{2 V_{\infty}} \frac{G(k)}{k}+c_{L_{\delta}} \delta \\
& \left.+\frac{\pi c}{2 V_{\infty}}\left(\frac{\dot{\alpha}}{\cos \Lambda}-W_{x t} \tan \Lambda-\Theta_{t}-\frac{W_{t t}+e_{m} \Theta_{t t}}{V_{\infty} \cos \Lambda}\right)\right] q_{\infty} \cos ^{2} \Lambda c-m g-m W_{t t}+m e_{c g} \Theta_{t t}
\end{aligned}
$$

These equations describe the wing bending and torsion deflections due to aerodynamic forces and moments. Using the Galerkin's method [15], the bending and torsional deflections can be approximated by the method of separation of variables as

$$
\begin{aligned}
\Theta(x, t) & =\sum_{i=1}^{n} \theta_{j}(t) \Psi_{j}(x) \\
W(x, t) & =\sum_{i=1}^{n} w_{j}(t) \Phi_{j}(x)
\end{aligned}
$$


where $\theta_{j}(t)$ and $w_{j}(t)$ are the generalized coordinates, and $\Psi_{j}(x)$ and $\Phi_{j}(x)$ are the assumed normalized eigenfunctions of the $j$-th bending and torsion aeroelastic modes, respectively, $j=1,2, \ldots, n$.

The normalized eigenfunctions are given by

$$
\begin{gathered}
\Psi_{j}(x)=\sqrt{2} \sin \frac{(2 j-1) \pi x}{2 L} \\
\Phi_{j}(x)=\cosh \left(\beta_{j} x\right)-\cos \left(\beta_{j} x\right)-\frac{\cosh \left(\beta_{j} L\right)+\cos \left(\beta_{j} L\right)}{\sinh \left(\beta_{j} L\right)+\sin \left(\beta_{j} L\right)}\left[\sinh \left(\beta_{j} x\right)-\sin \left(\beta_{j} x\right)\right]
\end{gathered}
$$

where $\beta_{j} L=1.87510,4.69409, \ldots$ is the eigenvalue of the $j$-th bending mode of a uniform cantilever beam, and the eigenfunctions $\Psi_{j}(x)$ and $\Phi_{j}(x)$ satisfy the orthogonal condition

$$
\int_{0}^{L} \Psi_{i}(x) \Psi_{j}(x) d x=\int_{0}^{L} \Phi_{i}(x) \Phi_{j}(x) d x= \begin{cases}L & i=j \\ 0 & i \neq j\end{cases}
$$

The weak-form integral expressions of the aeroelastic equations are obtained by multiplying the torsional and bending equations by $\Psi(x)$ and $\Phi(x)$ and then integrating over the wing span. Upon enforcing the zero boundary conditions at the two end points, the weak-form dynamic aeroelastic equations are obtained as

$$
\begin{aligned}
\sum_{j=1}^{n} \int_{0}^{L} \Psi_{i}^{\prime}\left\{\left[G J+E B_{1}\left(\gamma^{\prime}\right)^{2}\right] \theta_{j} \Psi_{j}^{\prime}-E B_{2} \gamma^{\prime} w_{j} \Phi_{j}^{\prime \prime}\right\} d x \\
+\sum_{j=1}^{n} \int_{0}^{L} \Psi_{i}\left[c c_{m_{a c}}+e c_{L_{\alpha}}\left(\frac{\alpha}{\cos \Lambda}+\frac{q x_{a c}}{V_{\infty} \cos \Lambda}-w_{j} \Phi_{j}^{\prime} \tan \Lambda-\theta_{j} \Psi_{j}-\frac{\dot{w}_{j} \Phi_{j}-e \dot{\theta}_{j} \Psi_{j}}{V_{\infty} \cos \Lambda}\right) F(k)\right] q_{\infty} \cos ^{2} \Lambda c d x \\
+\sum_{j=1}^{n} \int_{0}^{L} \Psi_{i} e c_{L_{\alpha}}\left(\frac{\dot{\alpha}}{\cos \Lambda}-\dot{w}_{j} \Phi_{j}^{\prime} \tan \Lambda-\dot{\theta}_{j} \Psi_{j}-\frac{\ddot{w}_{j} \Phi_{j}-e \ddot{\theta}_{j} \Psi_{j}}{V_{\infty} \cos \Lambda}\right) \frac{c}{2 V_{\infty}} \frac{G(k)}{k} q_{\infty} \cos ^{2} \Lambda c d x \\
\quad+\sum_{j=1}^{n} \int_{0}^{L} \Psi_{i} \sum_{k=1}^{m}\left(\frac{c}{e} c_{m_{\delta_{k}}} c_{L_{\delta_{k}}}\right) \delta_{k} e q_{\infty} \cos ^{2} \Lambda c d x \\
+\sum_{j=1}^{n} \int_{0}^{L} \Psi_{i} \frac{\pi c e_{m}}{2 V_{\infty}}\left(\frac{\dot{\alpha}}{\cos \Lambda}-\dot{w}_{j} \Phi_{j}^{\prime} \tan \Lambda-\dot{\theta}_{j} \Psi_{j}-\frac{\ddot{w}_{j} \Phi_{j}-e \ddot{\theta}_{j} \Psi_{j}}{V_{\infty} \cos \Lambda}\right) q_{\infty} \cos ^{2} \Lambda c d x \\
\quad-\sum_{j=1}^{n} \int_{0}^{L} \Psi_{i} m g e_{c g} d x+\sum_{j=1}^{n} \int_{0}^{L} \Psi_{i} m k^{2} \ddot{\theta}_{j} \Psi_{j} d x-\sum_{j=1}^{n} \int_{0}^{L} \Psi_{i} m e_{c g} \ddot{w}_{j} \Phi_{j} d x=0
\end{aligned}
$$

$$
\begin{aligned}
& \sum_{j=1}^{n} \int_{0}^{L} \Phi_{i}^{\prime \prime}\left(-E B_{2} \gamma^{\prime} \theta_{j} \Psi_{j}^{\prime}+E I_{y y} w_{j} \Phi_{j}^{\prime \prime}\right) d x \\
&- \sum_{j=1}^{n} \int_{0}^{L} \Phi_{i} c_{L_{\alpha}}\left(\frac{\alpha}{\cos \Lambda}+\frac{q x_{a c}}{V_{\infty} \cos \Lambda}-w_{j} \Phi_{j}^{\prime} \tan \Lambda-\theta_{j} \Psi_{j}-\frac{\dot{w}_{j} \Phi_{j}-e \dot{\theta}_{j} \Psi_{j}}{V_{\infty} \cos \Lambda}\right) F(k) q_{\infty} \cos ^{2} \Lambda c d x \\
& \quad-\sum_{j=1}^{n} \int_{0}^{L} \Phi_{i} c_{L_{\alpha}}\left(\frac{\dot{\alpha}}{\cos \Lambda}-\dot{w}_{j} \Phi_{j}^{\prime} \tan \Lambda-\dot{\theta}_{j} \Psi_{j}-\frac{\ddot{w}_{j} \Phi_{j}-e \ddot{\theta}_{j} \Psi_{j}}{V_{\infty} \cos \Lambda}\right) \frac{c}{2 V_{\infty}} \frac{G(k)}{k} q_{\infty} \cos ^{2} \Lambda c d x \\
&-\sum_{j=1}^{n} \int_{0}^{L} \Phi_{i} \sum_{k=1}^{m} c_{L_{\delta_{k}}} \delta_{k} q_{\infty} \cos ^{2} \Lambda c d x-\sum_{j=1}^{n} \int_{0}^{L} \Phi_{i}\left(\frac{\dot{\alpha}}{\cos \Lambda}-\dot{w}_{j} \Phi_{j}^{\prime} \tan \Lambda-\dot{\theta}_{j} \Psi_{j}-\frac{\ddot{w}_{j} \Phi_{j}-e \ddot{\theta}_{j} \Psi_{j}}{V_{\infty} \cos \Lambda}\right) q_{\infty} \cos ^{2} \Lambda c d x \\
& \quad+\sum_{j=1}^{n} \int_{0}^{L} \Phi_{i} m g d x+\sum_{j=1}^{n} \int_{0}^{L} \Phi_{i} m \ddot{w}_{j} \Phi_{j} d x-\sum_{j=1}^{n} \int_{0}^{L} \Phi_{i} m e_{c g} \ddot{\theta}_{j} \Psi_{j} d x=0
\end{aligned}
$$

The resultant matrix equation is obtained as

$$
M(k) \ddot{x}_{e}+C(k) \dot{x}_{e}+K(k) x_{e}+H(k) x_{a}=F+G \delta
$$


where $x_{e}=\left[\begin{array}{llllllll}\theta_{1} & \theta_{2} & \cdots & \theta_{n} & w_{1} & w_{2} & \cdots & w_{n}\end{array}\right]^{\top}$ is an elastic state vector of the generalized coordinates, $x_{a}=$ $\left[\begin{array}{ccc}\alpha & \dot{\alpha} & q\end{array}\right]^{\top}$ is an aerodynamic state vector of the angle of attack and pitch rate, $\delta=\left[\begin{array}{llll}\delta_{1} & \delta_{2} & \cdots & \delta_{n}\end{array}\right]^{\top}$ is a control vector of the flap deflections, $M$ is the reduced-frequency dependent generalized mass matrix, $C$ is the generalized damping matrix, $K$ is the generalized stiffness, $H$ is the generalized aerodynamic coupling matrix, $F$ is the generalized force vector, and $G$ is the generalized force derivative vector due to the flap deflections.

\subsubsection{Static Wing Deflection and Aeroelastically Tailored As-Built Wing Shape}

The aeroelastic equation includes both the static and dynamic components of the bending and torsional deflections. The static wing shaping control equation is obtained as

$$
K x_{e}+H x_{a}=G \delta
$$

The aircraft's optimal wing shape is designed to be maintained at all times during cruise by wing shaping control flap. To achieve a desired wing shape, the flap must be deflected to change the aerodynamic forces and moments acting on the wing so as to cause the wing to deflect to the desired wing shape. To determine the flap deflection requirements, the static wing shaping control equation can be written for a desired wing shape as

$$
K_{d} x_{d}+H x_{a}=G \delta_{d}
$$

where the subscript $d$ denotes the quantities associated with the desired wing shape.

Then subtracting these equations yields

$$
K_{d} x_{d}-K x_{e}=G \delta
$$

Using a Moore-Penrose pseudo-inverse method, $\delta$ can be computed as

$$
\delta=R G^{\top}\left(G R G^{\top}\right)^{-1}\left(K_{d} x_{d}-K x_{e}\right)
$$

where $R>0$ is a weighting matrix.
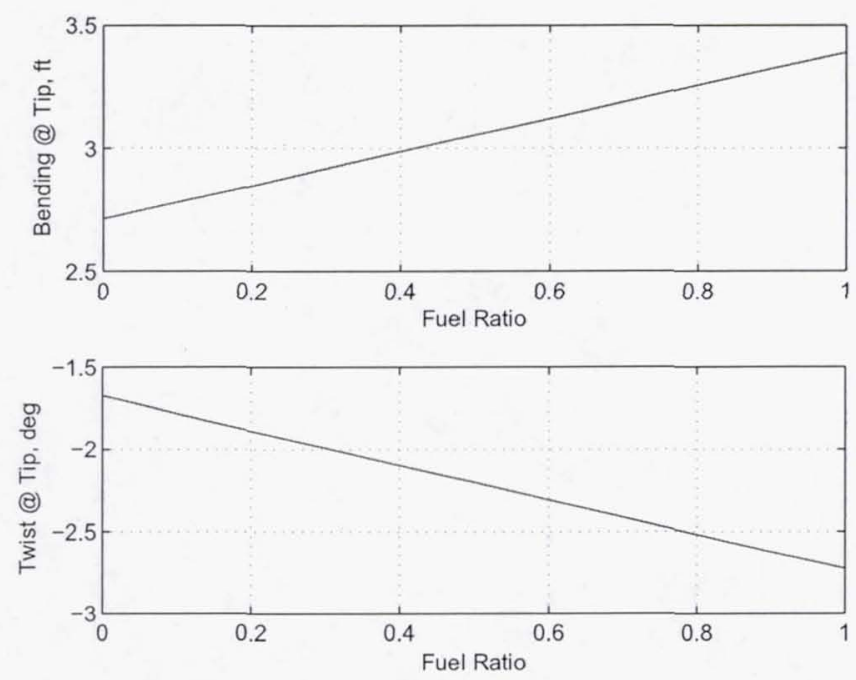

Figure 44 - Wing Tip Bending Deflection and Twist

The wing tip bending and torsion deflections as a function of fuel burn is shown in Figure 44 . It can be seen that the wing tip bending and torsional deflections decrease linearly with fuel burn. The deflections are maximum with the fuel tank full. The $50 \%$ fuel point is where the wing shape reaches its optimal drooped wing shape under the 1-g loading. Thus, the as-built wing shape would have an additional negative downward bending deflection of $3.0554 \mathrm{ft}$ and an additional positive nose-down twist of $2.2040^{\circ}$. This aeroelastic tailoring allows the jig-shape (as-built) wing shape to take on the desired wing shape in-flight under aerodynamic loading. The optimal and jig-shape drooped wing shape and twist are shown in Figures 45 and 46. 


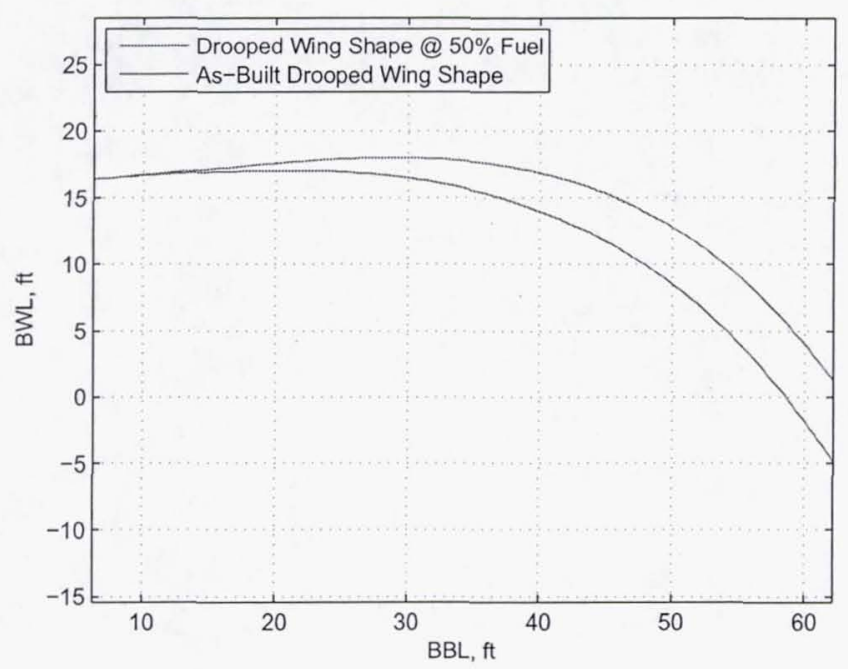

Figure 45 - Optimal and Jig-Shape Drooped Wing Shapes

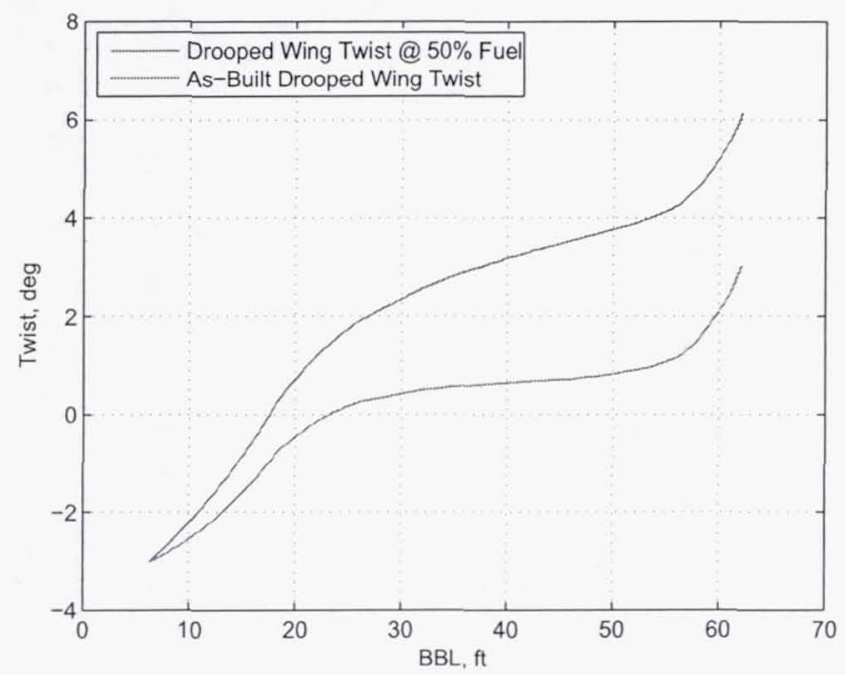

Figure 46 - Optimal and Jig-Shape Drooped Wing Twists

\section{Development of the Variable Camber Continuous Trailing Edge Flap Sys- tem}

Following the initial study conducted in 2010, Boeing and NASA are conducting a joint follow-on study program funded by the NASA Subsonic Fixed Wing (SFW) / Fixed Wing (FW) project to design a wing flap system that will provide mission-adaptive lift and drag performance for future transport aircraft having light-weight, flexible wings [18]. This Variable Camber Continuous Trailing Edge Flap (VCCTEF) system offers a lighter-weight lift control system having two performance objectives: (1) an efficient high lift capability for take-off and landing, and (2) reduction in cruise drag through control of the twist shape of the flexible wing. This control system during cruise will command varying flap settings along the span of the wing in order to establish an optimum wing twist for the current gross weight and cruise flight condition, and continue to change the wing twist as the aircraft changes gross weight and 
cruise conditions for each mission segment. Design weight of the flap control system is being minimized through use of light-weight shape memory alloy (SMA) actuation augmented with electric actuators. The VCCTEF program is developing better lift and drag performance of flexible wing transports with the further benefits of lighter-weight actuation and less drag using the variable camber shape of the flap.

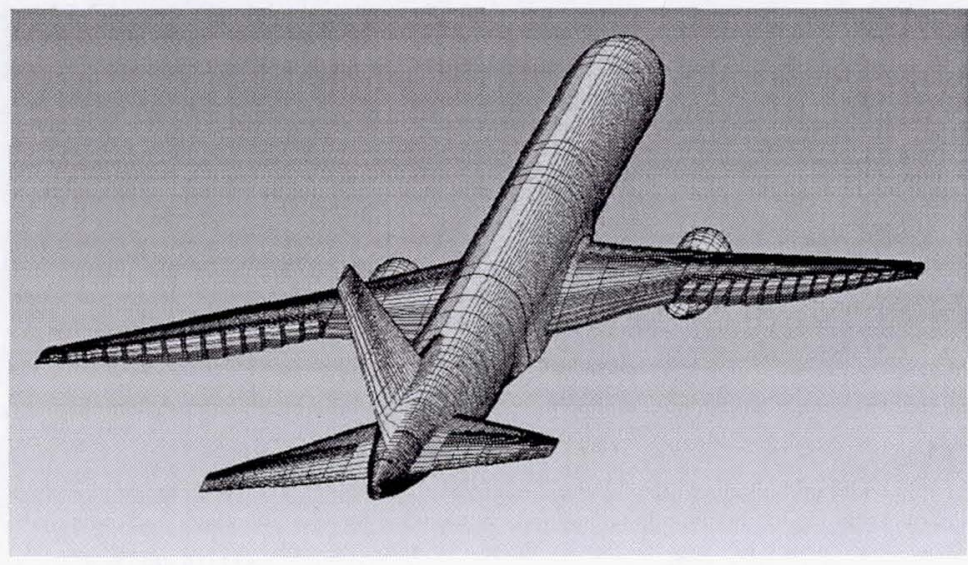

Figure 47 - GTM with VCCTEF

The flap is divided into 14 sections attached to the outer wing and 3 sections attached to the inner wing. Each 24-inch section has three camber flap segments that can be individually commanded. These camber flaps are joined to the next section by a flexible and supported material (shown in blue) installed with the same shape as the camber and thus providing continuous flaps throughout the wing span with no drag producing gaps.

Using the camber positioning, a full-span, low-drag, high-lift configuration can be activated that has no drag producing gaps and a low flap noise signature. This is shown in Figure 48.

To further augment lift, a slotted flap configuration is formed by an air passage between the wing and the inner flap that serves to improve airflow over the flap and keep the flow attached. This air passage appears only when the flaps are extended in the high lift configuration.

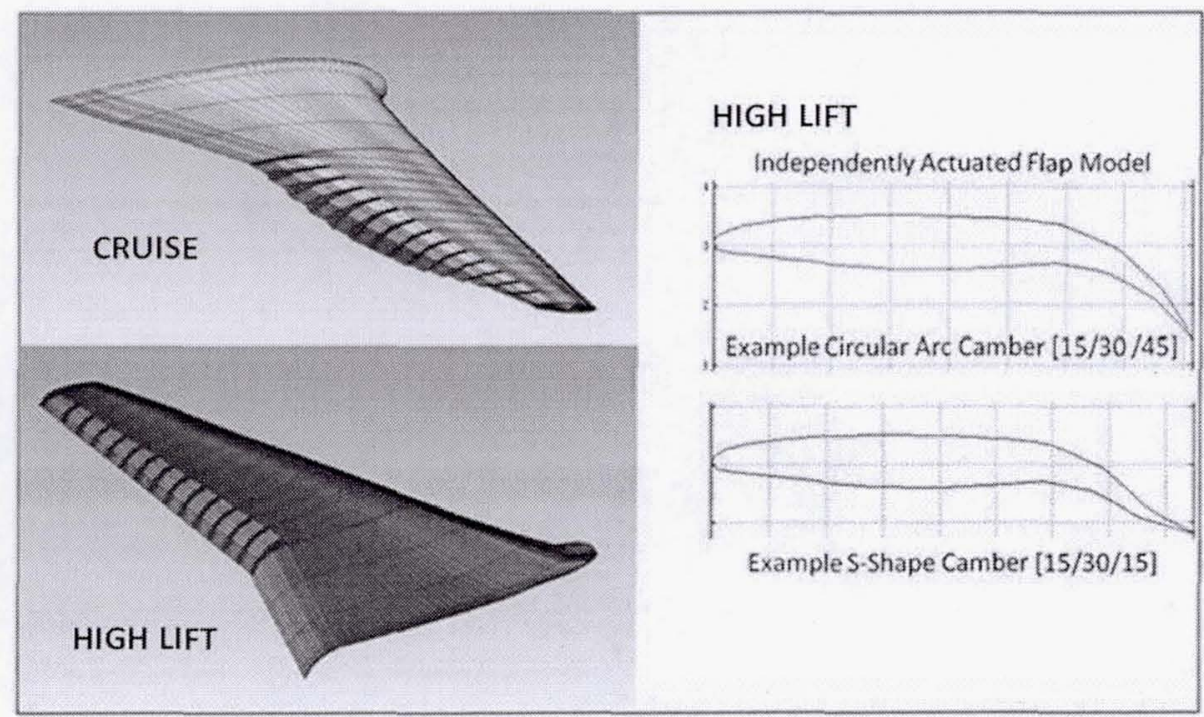

Figure 48. Cruise and High Lift VCCTEF Configurations

The flexible skin materials that cover the spanwise camber flap sections create constraints to the flap deflections. These constraints impose a certain relative flap deflection between any two adjacent spanwise flap sections. An 
unconstrained optimization is conducted first. Then, relative deflection constraints of $1^{\circ}$ and $2^{\circ}$ are added to the optimization. The unconstrained optimization results serve as upper limits of drag optimization with relative deflection constraints.

The results of the optimization are shown in Table 9.

\begin{tabular}{|c|c|c|c|}
\hline & Unconstrained & $2^{\circ}$ Constraint & $1^{\circ}$ Constraint \\
\hline \hline $80 \%$ Fuel Loading & $2.64 \%$ & $2.38 \%$ & $1.99 \%$ \\
\hline $50 \%$ Fuel Loading & $3.64 \%$ & $3.26 \%$ & $1.23 \%$ \\
\hline 20\% Fuel Loading & $9.88 \%$ & $5.71 \%$ & $3.24 \%$ \\
\hline
\end{tabular}

Table 9. Preliminary Induced Drag Optimization for Rigid Wing with Circular Arc Camber VCCTEF

The largest drag reduction is noted with $20 \%$ fuel loading. For $2^{\circ}$ relative deflection constraints, the drag reduction decreases to $5.71 \%$ from $9.88 \%$. There is a significant penalty in drag reduction benefit as the relative deflection constraints drop below $2^{\circ}$. This suggests that the flexible skin materials should be designed to allow at least $2^{\circ}$ flexibility.

It should be noted that these preliminary results only indicate qualitatively that drag reduction can indeed be achieved with the VCCTEF. Additional optimization work that includes effects of aeroelasticity and additional configuration variables will be conducted in the near future to estimate more precisely the potential drag reduction benefit of the VCCTEF.

\section{Discussion}

The optimization study has identified some potentially promising concepts such as the drooped wing shape and inflected wing shape. The potential drag reduction appears significant. However, this drag reduction may be associated mostly with lift-induced drag. It is still unclear how much changes in parasitic drag associated with these concepts would be. Therefore, more work would be needed to verify the potential drag reduction benefits of these concepts. High-fidelity CFD codes that capture viscous and compressibility effects may be able to better shed light on the merits of these new concepts. Nonetheless, the results of this study do warrant further investigation.

In terms of new revolutionary concepts such as drooped wing shapes if their drag reduction benefits can be verified, current constraints in aircraft development capabilities may preclude such concepts to be adopted due to potential technical challenges in many different areas. One obvious area is materials and structure. To accommodate such a wing shape, radical changes in material choices and structural design processes would have to occur. With wing shaping control design, cyclic loadings on aircraft wings during cruise would potentially result in fatigue life issues. Advances in composites material development may be able to address some of the challenges in materials and structure.

The fuel management is yet another technical challenge as fuel can no longer be gravity-fed, thus necessitating auxiliary equipment which can add weight that in turn can offset any drag reduction benefits.

Another obvious challenge with the drooped-wing aircraft concept is ground clearance. Figure 49 shows that the tip of the aeroelastic tailored as-built drooped wing shape is below BWL 0, which implies that the wing tip would touch the ground. Folded wing concepts such as those used in many World War II vintage aircraft could be used to increase ground clearance for take-off and landing. However, folded wing mechanisms will undoubtedly add weight back to the vehicle which will negate the benefit of drag reduction.

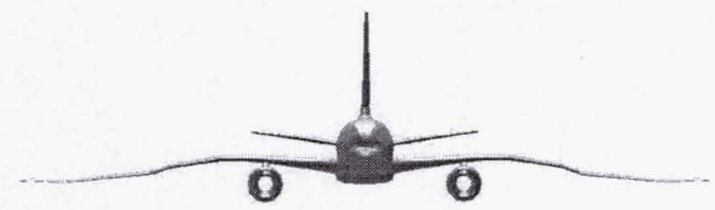

Figure 49 - Folded Wing Configuration for Take-Off and Landing

The inflected-wing aircraft concept may stand a better chance for any technology adoption without major radical changes to the current aircraft development capabilities. Some technical challenges may still be encountered with these configurations as with any new aircraft configuration no matter how feasible it may appear. 
Wing shaping control appears to have a potential drag reduction benefit. The study shows that to harvest the potential of wing shaping control, configuration changes in high-lift devices would have to be a part of the wing shaping control strategy. Flap and slat devices inherently generate drag as they increase lift. Conventional flap and slat systems as used in the current generation aircraft are not aerodynamically efficient enough for drag-reducing control strategies like wing shaping control. The variable camber continuous trailing flap concept developed in this study does seem to offer a potential pay-off for drag reduction even when used in current generation aircraft as a mission-adaptive control device. Technical challenges would exist as the increase in the number of multiple segmented flaps that form a variable camber continuous flap surface would lead to increased complexity. The continuous trailing edge feature will pose a challenge in the current flap system design since it may require a more conformable material that can accommodate differential deflections across a flap segment.

Another observation is made with regards to a wing shaping control strategy using flap-type actuation devices in that aerodynamic efficiency, which is the lift-to-drag ratio, would dictate wing shaping control flaps to operate only in the positive (downward) flap deflections. For a positively cambered wing section, a positive flap deflection causes the airfoil camber to increase that in turn generates more lift. Any attendant drag increase as a result of lift increase would be more desirable than drag increase as a result of lift decrease, as would be the case with negative (upward) flap deflections.

Finally the issues of wing flexibility on vehicle stability cannot be ignored. No matter how aerodynamically efficient an aircraft would be, it would never fly if it is unstable. Flight control can be used to stabilize aeroelastic instability that may be associated with wing flexibility. However, a stable aircraft is always desirable for commercial aviation. Aeroelastic tailoring by properly distributing wing stiffness throughout the airframe may also improve stability margins of aeroelastic modes. The role of flight control is then relegated to stability augmentation which would reduce the demand on a flight control system. In any case, increased wing flexibility would result in more susceptibility to potentially severe responses to air turbulence and wind gusts. Flight control systems would need to be designed to provide flutter suppression, gust load alleviation, and load limiting capabilities. These flight control capabilities may become standard one day for aircraft flight control system design as the trend in aircraft design is moving toward a more flexible airframe design for increased performance and reduced fuel burn.

\section{Conclusions}

This study presents the findings of the NASA Innovation Fund project entitled "Elastically Shaped Air Vehicle Concept" conducted over a four-month period in 2010. Three emerging themes have been developed in support of meeting national and global aviation challenges of reducing fuel burn for present and future aviation systems. These themes are intertwined and promote the multi-disciplinary nature of the goal of drag reduction in current and future aircraft.

The first theme addresses the drag reduction goal through innovative vehicle configuration optimization. Two elastic wing concepts have been identified from this theme: a drooped wing shape and an inflected wing shape. The drooped wing shape is a truly biologically inspired wing concept that mimics a seagull wing and could achieve about $5 \%$ to $6 \%$ drag reduction, which is aerodynamically significant. The inflected wing concepts could achieve about $3 \%$ drag reduction. While providing less drag reduction benefits than the droop wing shape, the inflected wing shape could have a near-term impact since this concept can be readily developed within the current aircraft development capabilities.

The second theme addresses the drag reduction goal through a new concept of elastic wing shaping control. The multi-disciplinary nature of flight physics is appreciated with the recognition of the adverse effects of aeroelastic wing shape deflections on induced drag as a result of changes in wing lift distributions. By aeroelastically tailoring the wing shape with active control, a significant drag reduction benefit could be realized. A significant drag reduction for long-range cruises from elastic wing shaping control could be realized as has been shown in the study when compared with the drooped-wing aircraft configuration without elastic wing shaping control.

To realize the potential of the elastic wing shaping control concept, the third theme emerges that addresses the drag reduction goal through a new type of aerodynamic control surfaces. A variable camber continuous trailing edge flap concept has been developed in this study. Conventional aerodynamic control surfaces are discrete independent surfaces that cause geometric discontinuities at the trailing edge region. These discontinuities promote vorticities which result in drag rises and noise sources. The variable camber continuous trailing edge flap concept could provide a substantial drag reduction benefit over a conventional discrete flap system. Aerodynamic simulations show a drag reduction of over $50 \%$ could be achieved with the flap concept over a conventional discrete flap system. Moreover, elastic wing 
shaping control potential could only be realized with low drag aerodynamic control surfaces such as this flap concept in order to keep the incremental drag due to flap deflections as small as possible.

Research and technology development of new vehicle concepts to support the drag reduction goal requires a multidisciplinary approach that integrates knowledge domains across different disciplines to bring forth potential solutions that recognize the interactive nature of these disciplines in contributing to the common goal of drag reduction. This important aspect of research and technology development was recognized and a multi-disciplinary study was conducted to bring together a diverse set of disciplines in aerodynamics, optimization, structural analysis and aeroelasticity, flight dynamics and control, and system analysis in order to develop an integrated approach for future vehicle system design.

\section{Acknowledgment}

The authors wish to acknowledge NASA Innovative Partnerships Program for the support of this study under the NASA Innovation Fund Award in 2010 and NASA Aeronautics Research Mission Directorate for their technical support through the Subsonic Fixed Wing Project under the Fundamental Aeronautics Program. The technical execution of this project is contributed by NASA Ames Research Center, NASA Langley Center, Boeing Research and Technology in St. Louis, and California State University Sacramento.

\section{References}

[1] Nguyen, N., "Elastically Shaped Future Air Vehicle Concept," NASA Innovation Fund Award 2010 Report, October 2010, submitted to NASA Innovative Partnerships Program, http://ntrs.nasa.gov/archive/nasa/casi.ntrs.nasa.gov/20110023698_2011024909.pdf

[2] Warwick, G., "Flying Greener, on the Wings of a Gull," Aviation Week \& Space Technology, Leading Edge AW\&ST on Technology, May 26, 2011.

[3] Jordan, T., Langford, W., Belcastro, C., Foster, J., Shah, G., Howland, G., and Kidd, R., "Development of a Dynamically Scaled Generic Transport Model Testbed for Flight Research Experiments," AUVSI Unmanned Unlimited, Arlington, VA, 2004.

[4] Stanford University, AA241, "Aircraft Design: Synthesis and Analysis," http://adg.stanford.edu/aa241/structures/weightstatements.html

[5] Nguyen, N., Nelson, A., and Pulliam, T., "Damage Adaptive Control System Research Report," Internal NASA Report, April 2006.

[6] Miranda, L., Elliot, R., and Baker, W., "A Generalized Vortex Lattice Method for Subsonic and Supersonic Flow Applications," NASA CR-2865, 1977.

[7] Aftosmis, M., Berger, M., and Melton, J., "Robust and Efficient CartesianMesh Generation for Component- Based Geometry," AIAA Journal, Vol. 36, No. 6, 1998, pp. 953-960.

[8] Nemec, M., Aftosmis, M., Murman, S., and Pulliam, T., "Adjoint Formulation for an EmbeddedBoundary Cartesian Method," AIAA 2005-0877, 43rd AIAA Aerospace Sciences Meeting, Reno, NV, Jan. 2005. (Also NASA Technical Report NAS-05-008)

[9] Nemec, M., Aftosmis, M., and Wintzer, M., Adjoint-Based Adaptive Mesh Refinement for Complex Geometries, AIAA 2008-0725, 46th AIAA Aerospace Sciences Meeting, Reno, NV, Janurary 2008.

[10] Roach, J., "Seagulls May Inspire New Airplane Wings," http://news.nationalgeographic.com/news/2006/10/061024seagull-wings.html, National Geographic News, October 24, 2006.

[11] The Boeing Company, "The Active Aeroelastic Wing Flight Research Program (The X-53) Final Report,",Vol. I and II, AFRL-VA-WP-TR-2005-3082, October 2005.

[12] Theodorsen, T. and Garrick, I., "Mechanism of Flutter - a Theoretical and Experimental Investigation of the Flutter Problem", NACA Report 685, 1940. 
[13] Theodorsen, T, "General Theory of Aerodynamic Instability and the Mechanism of Flutter", NACA Report 496, 1935.

[14] Roskam and Lan, Airplane Aerodynamics and Performance, DARcorporation, 1997.

[15] Hodges, D. and Pierce, G., Introduction to Structural Dynamics and Aeroelasticity, Cambridge University Press, 2002.

[16] Nguyen, N., "Integrated Flight Dynamic Modeling of Flexible Aircraft with Inertial Force-PropulsionAeroelastic Coupling," AIAA-2008-194, 46th AIAA Aerospace Sciences Meeting, Reno, NV, Janurary 2008.

[17] Houbolt, J. and Brooks, G., "Differential Equations of Motion for Combined Flapwise Bending, Chordwise Bending, and Torsion of Twisted Nonuniform Rotor Blades," NACA Technical Note 3905, February 1957.

[18] Urnes, J., Nguyen, N., Ippolito, C., Totah, J., Trinh, K., Ting, E., “A Mission-Adaptive Variable Camber Flap Control System to Optimize High Lift and Cruise Lift-to-Drag Ratios of Future N+3 Transport Aircraft," 51st AIAA Aerospace Sciences Meeting, Grapevine, TX, Janurary 2013. 